\title{
The Progressive Smart Grid System from Both Power and Communications Aspects
}

\author{
Chun-Hao Lo, Student Member, IEEE, and Nirwan Ansari, Fellow, IEEE
}

\begin{abstract}
The present electric power system structure has lasted for decades; it is still partially proprietary, energyinefficient, physically and virtually (or cyber) insecure, as well as prone to power transmission congestion and consequent failures. Recent efforts in building a smart grid system have focused on addressing the problems of global warming effects, rising energy-hungry demands, and risks of peak loads. One of the major goals of the new system is to effectively regulate energy usage by utilizing the backbone of the prospectively deployed Automatic Meter Reading (AMR), Advanced Meter Infrastructure (AMI), and Demand Response (DR) programs via the advanced distribution automation and dynamic pricing models. The function of the power grid is no longer a system that only supplies energy to end users, but also allows consumers to contribute their clean energy back to the grid in the future.

In the meantime, communications networks in the electric power infrastructure enact critical roles. Intelligent automation proposed in smart grid projects include the Supervisory Control And Data Acquisition/Energy Management Systems (SCADA/EMS) and Phasor Management Units (PMU) in transmission networks, as well as the AMR/AMI associated with field/neighborhood area networks (FAN/NAN) and home area networks (HAN) at the distribution and end-use levels. This article provides an overview of the essentials of the progressive smart grid paradigm and integration of different communications technologies for the legacy power system. Additionally, foreseeable issues and challenges in designing communications networks for the smart grid system are also rigorously deliberated in this paper.
\end{abstract}

Index Terms-Electric power system, smart grid, demand response, renewable energy, distributed generation, micro grids, standards, wireline and wireless communications, technologies integration, interoperability, coexistence, IP adoption.

\section{INTRODUCTION}

$\mathbf{E}$ LECTRICITY has been an indispensable resource for living. Power plants, generators, batteries, and large energy storage have been vastly manufactured and built from time to time to ensure that electricity would geographically serve every end point of territories and regions throughout the world. Today, typically in the United States, there are more than 10,000 central power plants, 5,600 distributed generation (DG) facilities, 200,000 miles of greater than $230 \mathrm{kV}$ high voltage transmission lines, 14,000 transmission substations, and 4,500 switching centers, operated by over 3,000 independent companies regulated by public utility commissions across the states

Manuscript received 17 February 2011; revised 2 May 2011

C.-H. Lo and N. Ansari are with the Advanced Networking Laboratory at the Department of Electrical and Computer Engineering, New Jersey Institute of Technology, Newark, NJ, 07102 USA (e-mail:CL96@njit.edu and Nirwan.Ansari@njit.edu).

Digital Object Identifier 10.1109/SURV.2011.072811.00089 and regions [1]-[4]. The statistics tend to swell further in view of the inevitable population explosion and increasing need for energy use. Along with the climbing quantity of electric power components, the phenomena of declining reliability and power quality is becoming a burden to the society. The world is hot, flat and crowded [5] because the temperature is rising in the past thirty years, the number of middle-class around the world is expanding, and the world population is dramatically growing.

Electricity is conventionally generated in the form of centralized electric power systems invented predominantly during the industrial revolution. The current structure of the electric power system particularly in U.S. nearly remains unchanged as compared to the one built more than a century ago [5][7]. A minor improvement to the existing system has been upgrades only on the material types and construction designs used for transformers, transmission lines, poles, and insulators [8]. Problematically, the power grid is mainly fossil fuelsbased, constrained to legacy infrastructure and functionalities, as well as subject to transmission congestion and cascading failures. The smart grid design originally proposed by the government is going to broadly address a list of problems in the legacy power system, and to advance the grid on an unprecedented scale in the next twenty years or so.

The legacy has considerably experienced limitation of the market and economic growth and a number of desperate circumstances that could jeopardize the stability of the system operation and environment. The fundamental triggers for the smart grid are attributed to the following:

- Dirty power plants as well as climate change: more than $80 \%$ of resources used to produce energy throughout the world today is fossil fuels, i.e., non-renewables [9]. Burning fuels were economical, and environment was historically not a concern. Unfortunately at present, the greenhouse gas effect has likely caused climate change and environmental impact.

- Centralized power generation as well as aging infrastructure: the centralization and aging problems would endanger the power system operation from a long-term perspective. The frequency of instigating peak load and power outage is likely to increase. It will become harder to restore and prevent from happening as the demand for energy exacerbates rapidly.

- Rising energy demands: along with the world population growth, there is an increase in pace of industrialization in developing countries, such as China and India. Without improving responsiveness from consumers as well as the 
system, rising demand may not be easily controlled at later times.

- Climbing fuel costs: the phenomenon of manipulation of energy supplies and competition over energy resources has impelled nations to reassess the price of dependence on imported energy.

- Deteriorating reliability: the current electricity system is claimed to be $99.97 \%$ reliable but still allows for power outages and interruptions [10]. It would be more difficult, uneconomical, and inefficient to keep up with the pace of rising demands as well as fuel costs.

- Utility monopoly: utility is the only energy supplier to consumers. Consumers are asked to pay for their utilities without knowing how much energy they have used and how much money they have been charged until the end of the billing cycle.

- Limitation of innovation and modernization: the power grid without changing its current infrastructure and operation method can hardly benefit utilities and society in the long term. For instance, continuously depending on fossil fuels and staying in the centralized form could restrict or impede the growth of the system to meet the energy demand.

In addition to the aforementioned considerable issues, the power grid infrastructure is further vulnerable to 1) natural disasters, severe weather, and accidents, 2) small mistakes, which could consequently cause bigger problems, and 3) physical and cyber attacks. Nonetheless, the smart grid plan entails several obstacles: regulatory factors, financial concerns, and return on investment; utilities are hesitating to choose the right technologies for the smart grid. Meanwhile, consumers' desire to partake in the smart grid will depend upon how much benefits, easiness, and security as well as privacy the program can deliver. Without efforts from either utilities or consumers, the smart grid cannot be fully achieved.

Unidirectional flow of communications and energy is another major issue to be solved in today's grid: there is no interaction between utilities and consumers because hightech power facilities, microprocessors, and communications sensors, were not available at the time the grid was built. Consumers so far have no way of knowing how much energy they have consumed or have not but accidentally wasted through leakage until the electricity bill is received. Most of the utilities are not capable of tracking and measuring the power usage by end-use sectors in real-time because of legacy constraints as well as lack of tools and equipment, e.g., conventional electricity meters or some automatic meter reading (AMR) meters that can only perform one-way communications. A smart grid will not only enable real-time tracking and measurements of utilities, but useful programs and features can be further developed and provided to customers. In return, customers are willing to interact with utilities by making their own choices on how to use energy based on time-of-use (TOU) price via user interfaces in home area networks (HAN). Moreover, utilities have been supplying power to customers but not the other way around. By enabling reverse power supply from customers to utilities, the smart grid fosters a close buyers-and-sellers relationship between customers and utilities. A two-way flow of power and communications as well as plug-and-play capabilities are the ultimate goal of the smart grid that will enable various technologies to supply, deliver, and use the resources reliably, efficiently, and securely.

In order to achieve the goals, the key driving forces to a successful smart grid evolution and revolution entail: 1) continual exploration for new sources of energy and investment in renewable resources development; 2) advancement and sustainability of facilities and equipment throughout the electric power system; 3) extension and placement of distributed energy capacities, renewables, and storage where loads are served; 4) innovation of information and communications technology (ICT) integrated with the legacy infrastructure; and 5) motivation of customers participation.

The remainder of this article is organized as follows: In Section II, smart grid is demonstrated in a what-why-who-andhow format to describe its definition, purpose, key players, and strategies. We will then provide a list of reports on recent smart grid projects as well as activities that have been initiated along with survey results. Section III discusses a list of capabilities and functionalities for smart grid to achieve. Section IV introduces a variety of potential technologies and networks likely to be employed in the smart grid system. We will further discuss the issues of integrating the diversities and several major challenges of designing the smart grid communications networks. Finally, Section V concludes this article. Throughout the literature, the terms energy, power, electricity, and electric power are interchangeably used, and so are customer and consumer.

\section{From Power Perspective: Tomorrow's Smarter AND Cleaner POWER GRID Infrastructure}

\section{A. What A Smart Grid Is}

An electric power system is fundamentally composed of three operational sectors: generation, transmission, and distribution. Generation is a process of producing power at power plants by employing numerous types of energy resources, e.g., fossil fuels. Examples of fossil fuels are typically coal, petroleum, (aka crude oil or liquids), and natural gas. Transmission and distribution involve delivering generated power from high/medium-voltage (HV/MV) to low-voltage (LV) through (step-up/step-down) transformers and eventually reaching the end-use sectors. Series of actions are regulated by a set of standards (e.g., IEEE, IEC, ANSI, CIP) [11]-[13] and a batch of data collection and automation [14] in order to operate the entire system consistently. Transmission lines connecting the various power facilities throughout the system construct the so-called power grid.

Power facilities mainly comprise power generators, stations, substations, and control centers in which electrical components $^{1}$ are built from multiple vendors. Control systems installed in the power grid for wide-area monitoring and control as well as substation and distribution automation are typically the conventional Supervisory Control And Data Acquisition and Energy Management Systems (SCADA/EMS) as well as the sophisticated synchrophasors Phasor Management Units

\footnotetext{
${ }^{1}$ Examples of electrical components include conductors, protective devices, capacitors, reactors, intelligent electronic devices (IEDs), programmable logic controllers, and remote terminal units.
} 
TABLE I

ANALOGOUS DEFINITIONS IN DESCRIBING THE CONCEPT OF THE SMART GRID.

\begin{tabular}{|c|c|}
\hline \multicolumn{2}{|r|}{ WE ARE BUILDING A SMART GRID THAT... } \\
\hline Ref. & Definition \\
\hline [16] & $\begin{array}{l}\text { Entails a transformation to an information-enabled and a highly interconnected network between electricity consumers and electric } \\
\text { suppliers embracing transmission, distribution, and generation. }\end{array}$ \\
\hline [17] & Creates the platform for deploying smart technologies that improve load management and demand response. \\
\hline [18] & $\begin{array}{l}\text { Will make the nation's power transmission system more efficient, encourage renewable energy sources, and give consumers better } \\
\text { control over their electricity usage and costs. }\end{array}$ \\
\hline [19] & $\begin{array}{l}\text { Is a multibillion dollar modernization of the electricity grid that involves supporting real-time, two-way digital communications } \\
\text { between electric utilities, and their increasingly energy-conscious customers. }\end{array}$ \\
\hline [20] & $\begin{array}{l}\text { Is a collection of next generation power delivery concepts that includes new power delivery components, control and monitoring } \\
\text { throughout the power grid, and more informed customer options. }\end{array}$ \\
\hline [21] & $\begin{array}{l}\text { Is a modern, improved, resilient, and reliable electric grid that provides for environmental stewardship, is secure, cost effective, } \\
\text { and is a predominant driver to economic stability and/or development. }\end{array}$ \\
\hline [22] & $\begin{array}{l}\text { Is a modern electric system. It uses sensors, monitoring, communications, automation and computers to improve the flexibility, } \\
\text { security, reliability, efficiency, and safety of the electricity system. }\end{array}$ \\
\hline [23] & an infrastructure that puts the emphasis on active rather than passive control. \\
\hline
\end{tabular}

\section{Application Layer}

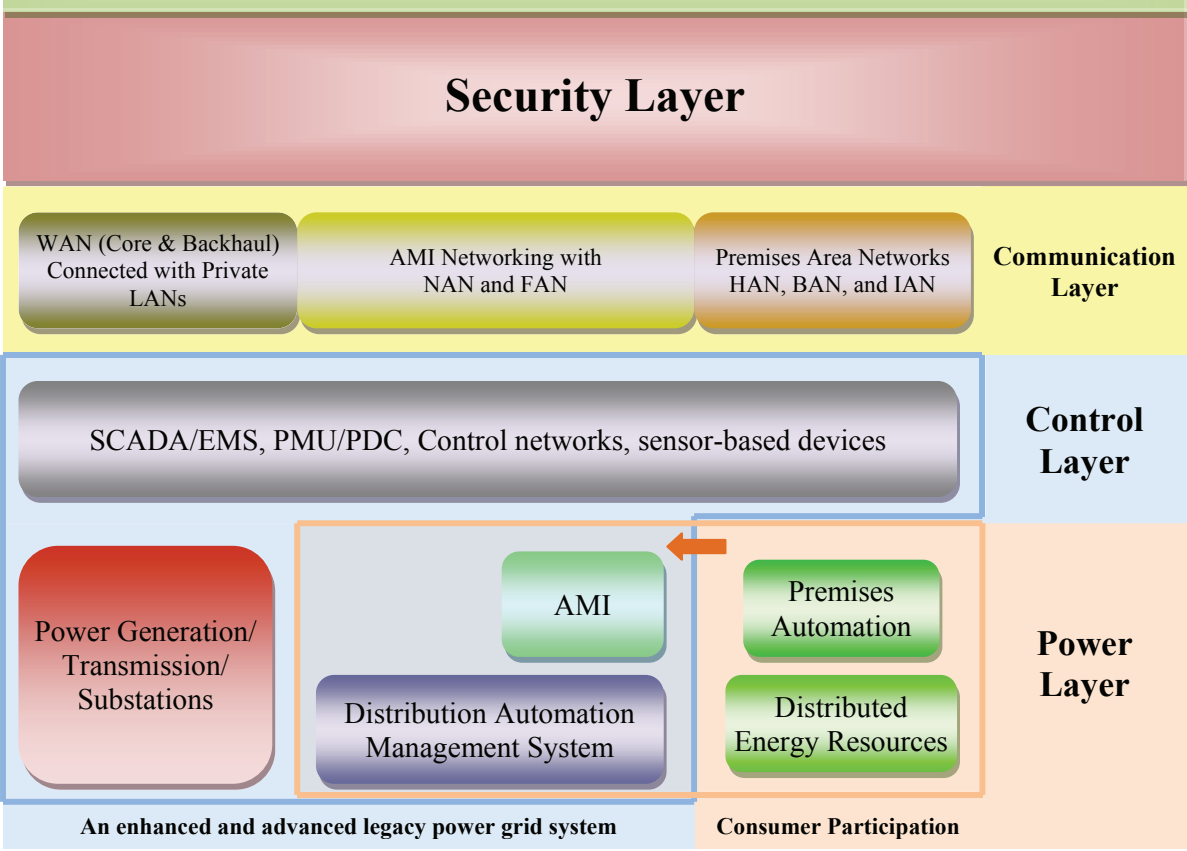

Fig. 1. The layered Smart Grid paradigm.

and Phasor Data Concentrators (PMU/PDC). SCADA typically measures voltage, current, and frequency once every few seconds in a format medically analogous to the X-ray, whereas PMU is capable of delivering more and complex samples per second likened to the MRI [10], [15]. Data flows in the system carry various power factors and measurements including substation and feeder monitoring, volt var control, FDIR (fault detection, isolation and restoration/recovery), transformer and motor temperatures, as well as the status of breakers, relays, and switchgear. The majority of communications protocols or simply protocols used in data exchange and management among substations are proprietary and regulated by utilities, municipalities, or regulators. Communications in the HV/MV transmission grid systems currently have been administered under advanced and sophisticated control and monitoring as well as computing tools.

The legacy power grid infrastructure particularly in the United States has mostly been constructed in a centralized radial tree-like topology. The formation is very vulnerable to attacks and failures because serious aftermath would affect multiple served regions if one single generation point collapses. In addition to the security issue, greenhouse gas emissions and power outage are recent major concerns.

Table I describes the smart grid blueprint with allied connotation. The reforming power grid system is likely referred to as the Third Industrial Revolution for energy. The modernization enables consumers engagement with demand response applications, distributed generation deployment (i.e., microgrids and 
virtual generation or buffers), as well as correspondingly intelligent distribution management system (DMS). The paradigm is illustrated in Figure 1 [24]. The smart grid is constructed in five dominant layers: power layer specifies smart power generation, conversion, transport, storage, and consumption; control layer involves smart sensors and actuators for data collection and conveyance as well as sensing and control; communication layer characterizes different roles to ensure accurate and effective data transmission; security layer assures data confidentiality, integrity, authentication, availability, and non-repudiation; and application layer supports decision intelligence with the aid of information technologies for various smart energy applications.

The concept of smart grid is not new. In fact, smart grid programs such as renewable energy deployments have been started in European countries as well as several regions in the United States approximately two decades ago. In the past few years, the demand response (DR) program has been initiated in some large industrial and commercial sectors [10]. Moreover, the remarkable Department of Energy's (DOE) smart grid demonstration projects in Olympic Peninsula, Washington have been successful in saving consumers' bills with the aid of energy DR management through smart thermostats and appliances on consumers' behalf [10]. Under the guidance of the small local smart grid projects in motion throughout the world and nations, a large-scale smart grid paradigm is no longer a mystery.

While activating the inclusion of the modernized distribution operation and participation of consumers, the smart grid will facilitate as well as consolidate substation and distribution automation [25]-[27], DMS [28], automatic meter reading and advanced meter infrastructure (AMR/AMI) [20], [27], [29]-[33], meter data management (MDM) [34], as well as demand side management (DSM) [35]. In order to meet the objectives, the smart grid will integrate the current stateof-the-art ICTs into the power infrastructure to help build smart control centers, smart substations, and smart transmission/distribution networks [36]. A number of imperative investigations are being conducted for the electrical power industry, e.g., optimizing the quality of energy delivery (e.g., free of line sags, disturbances, blackouts) and power transmission (or flow) control [37], as well as integrating DG, renewables, and storage [7], [38]. The layered architecture and integrated multi-tier network [24], [39] in the autonomous bidirectional smart grid system will show the significance of accommodating data acquisition technologies, data integration, broadband telecommunications, HAN, DSM, DG, as well as diversities of management, monitoring and services.

\section{B. The Purpose of The Smart Grid}

The following present several notable motivations for initiating the smart grid blueprint from a long-term investment perspective.

1) To Be Smarter: The intelligence of smart grid is to be self-coordination, -awareness, -healing, and -reconfiguration. In order to accomplish the requirements (to be discussed in Section III), the system must be effectively well organized such that its operations are carried out in an uncomplicated and controlled way. It always has the knowledge and understanding of the overall performance and status in real time throughout the entire grid based on information it receives and past experience it has encountered. Information is essentially categorized into vital messages for protection, which have to be transported at (near) real-time for crisis notification [8], [12], [14], and periodic (or less-critical) measurements for operation and information as well as continual monitoring that can be somewhat delayed or discarded momentarily. Both of them should be retrieved and utilized for regular reporting, midway adjustment, future strategic planning-predictionprevention, as well as pricing and billing for consumers energy usage. The system is able to know what and how to recover when abnormalities are discovered through the fully automated processes of fast-response detection and analysis. Eventually, the operation is automatically restored within a tolerable time.

Interestingly, the operation of the electric power system has similar characteristics found in the Internet [1]. Both of them have the same goal of delivering particular needs from the source to the destination over optimized routes and intermediate nodes by using strategic algorithms to avoid unendurable congestions and broken links. The similarities include

- Power plants, substations, control centers and end users versus service providers, terminals, intermediate and end nodes;

- Centralized infrastructure (designed to be decentralized in smart grid) in power system versus hybrid or ad-hoc topologies in communications networks;

- Power transmission capability versus data bandwidth capacity;

- Electricity versus communications data;

- Power transmission cables or lines versus data communications links.

Handling the power network system is like tackling performance problems in communications simultaneously. While the swiftness of power transmission is substantially dependent upon the advanced electric power facilities and technologies, data control and communications enact effective and conducive roles for the system to function smoothly. At the same time, the steadiness of signals transmission will rely upon how well the various communications technologies are interconnected and integrated.

The achievement of the smart grid will essentially be determined by interoperability as well as environmental and social constraints. It further entails broad knowledge in analog and digital control, communications theory, dynamic optimization, as well as the auxiliary standards, agile algorithms, communications protocols, and information technologies.

2) To Boost the Deployment of Renewable Energy Sources $(R E S)$ : Despite air pollution (i.e., carbon footprint) produced by burning fossil fuels, the lower capital cost is much attractive with respect to an equivalent amount of energy that can be generated as compared to non-fossil fuels. The dilemma casts hesitations on many investors in investing in renewal energy and smart grid. Inevitably, the quintessence of global warming and climate change has been acid rain, glacial meltdown, natural disaster, and severe weather around the world. According to the U.S. Environmental Protection Agency (EPA) 
and Energy Information Administration (EIA), reports have revealed the unavoidable phenomena of rising temperature and swelling greenhouse gas emissions: $\mathrm{CO}_{2}$ (carbon dioxide), $\mathrm{CH}_{4}$ (methane), and $\mathrm{N}_{2} \mathrm{O}$ (nitrous oxide) [5], [9], [40]. The majority of primary gases is emitted considerably by human activities, fossil fuel, and automobile combustion, among which $20 \%$ of $\mathrm{CO}_{2}$ is emitted by the transportation sector and $40 \%$ by power generation [10]. While purifying combustion is an option to reduce limited amount of harmful pollution, investment in RES should be a wiser and preferable longterm strategy.

Energy is typically consumed by four end-use sectors. Throughout the world on average [9], 30\% of the total energy each is consumed by industrial and transportation, respectively, whereas $20 \%$ of total each is consumed by residential and commercial, respectively. In 2008, the ratios of energy use by fuel type are $36 \%$ for petroleum, $27 \%$ for coal, $23 \%$ for natural gas, $8 \%$ for hydroelectric and renewables, and $6 \%$ for nuclear. Coal and nuclear are predominantly used for driving power plants; natural gas is for residential and commercial; both natural gas and petroleum are for industrial; transportation is mostly powered by petroleum. The statistics from EIA have shown that the regions Asia and Oceania as well as countries United States, China, Russia, India, and Japan are currently on the top of the list in consuming more energy and producing more $\mathrm{CO}_{2}$ gas than others [9]. Most of developed countries tend to reduce coal-based energy consumption and $\mathrm{CO}_{2}$ emission to some extent by deploying renewables. In the meantime, the developing nations continue to demand for more resources and likely to produce more greenhouse gases.

$\mathrm{RESs}^{2}$ essentially comprise solar, wind, geothermal, water power, and biofuels. Although renewables produce little (when burning waste, for instance) or no waste and greenhouse gases, they are expensive to build, unpredictable (i.e., climate and geography dependent), and constrained to the requirement of wide-area deployment for better effects and performance. However, as technology improves and fuel cost rises, it is an opportunity to adopt renewables in the long term. There have been successful cases of investing in renewables worldwide, such as U.S. (e.g., California, Texas), Europe (e.g., Germany, UK, Spain, Italy), Australia, and Japan. Reports have shown an astonishing growth in wind and biomass in year 2008 and 2009 [41]: U.S. has taken over the lead from Germany in wind markets since 2008 with China standing right behind U.S., as well as Spain and India in the fourth and fifth, respectively, in 2009. On the side of solar PV market installations [42], U.S. was placed in third in both 2008 and 2009, while Spain and Germany were the first and second in 2008. In 2009, Germany has jumped back to the first place, Italy climbed to the second, Japan the forth right behind U.S., and Spain has fallen off the top list.

Notably, by virtue of a larger social vision of smart grid (i.e., higher smart grid IQ) into which many European utilities have tapped for quite some time [2], the national politics

\footnotetext{
${ }^{2}$ Examples for solar: photovoltaic and concentrated solar power (PV/CSP); water power: hydroelectric, tidal, wave, and ocean; biofuels: biomass, biogas, living materials, and waste.
}

and strategic plans for green energy development and implementation throughout its electric power system are examples to other nations. Several European countries have pledged to become free of fossil fuels completely, such as Denmark and Sweden. Likewise, Portugal is enthusiastically pursuing aggressive renewable portfolio targets [2].

Developing renewables and constructing energy conserving buildings at the same time are much necessary for the environmental reason, conservation goal, and next-generation modernization. Indeed, they have been the top items on the to-do list of the smart grid. In accordance with DOE, making the grid 5\% more efficient is equivalent to eliminating fuel and greenhouse gas emissions from 53 million cars [10]. Therefore, such evolution and effects should be rigorously considered. Further readings on the impacts of climate change associated with energy loads at end-use sectors and strategymaking for electric power industries can be found in [43] and [44].

3) To Augment the Efficiency of Generation, Transmission, and Usage for Energy: It is inevitable that the amount of energy generated will always be greater than that of energy consumed in order to avoid power outage ${ }^{3}$. Besides, power transmission loss in transmission and distribution networks has been a critical issue. Recall the power-loss theory: the farther distance the two end units (i.e., supplier and consumer) is, the larger loss the received power is. Prior to power transmission, energy loss initially occurs at power plants (especially conventional and nuclear) during the energy input-to-output conversion and operation. According to EIA's Annual Energy Review 2008 [9], approximately two-thirds of total energy input is lost in the thermaldynamical conversion at power plants. In addition, roughly $5 \%$ is further lost in power plant use and $7 \%$ is lost in the transmission and distribution journey before reaching the end-use sectors. Eventually, less than $30 \%$ of the total input energy is transported to customers. Furthermore, energy can be wasted on premises where lights and HVAC (Heating, Ventilating, and Air Conditioning) remain $O N$ when no one is home, in the office (or building), or part of areas in these places. Besides, power leakage can further decrease energy utilization, which is likely to occur in older buildings where people are barely able to notice.

Conclusively, the efficiency of power transport and energy use has to be improved by making the electric power system smarter and by educating the consumers. If every household starts replacing one incandescent light bulb with a CFL bulb ${ }^{4}$, the conserved energy can be saved to light additional three million homes [10]. A transition from the traditionally centralized infrastructure to a decentralized form will likely mitigate the energy loss issue since energy sources are allocated close to the end-use consumers. The smart grid helps implement and interconnect the distributed energy resources (DER) ${ }^{5}$ to increase energy efficiency. Making the grid smart will need

\footnotetext{
${ }^{3}$ The redundant generated energy is called "reserve power" for maintenance and refueling purposes to sustain the system reliability.

${ }^{4} \mathrm{~A}$ compact fluorescent light (CFL) bulb consumes much lower power $(13 \mathrm{~W}, 19 \mathrm{~W}, 23 \mathrm{~W})$ and has longer rated life in comparison with the incandescent light bulb (60W, 75W, 100W).

${ }^{5}$ DERs include solar, wind, microturbines, combined heat and power (CHP), fuel cells, plug-in hybrid electric vehicles or pure electric vehicles (PHEV/EV), and energy storage
} 
disciplines, intelligent modeling, analyses, evaluation, and optimization to overcome these challenges.

4) To Shift and Customize Consumers' Energy Demands: The dramatic rising energy demand likely causes the peak load and power outage. Power equipment can be damaged and life-shortened by either over-generating the power at power plants when demand reaches the peak or inappropriately shutting down partial generators when demand is low. Hence, making the energy load as flat as possible over time is a straightforward solution. However, it may not be an easy task without the involvement of consumers. Therefore, the smart grid aims to empower consumers to be part of decision makers, help them learn to be responsible energy users, and incorporate them into the smart grid project throughout the power ecosystem.

In order to meet the state of stability, reliability, and consistency for power quality in the future, peak loads have to be reduced and loads are balanced on a daily (even hourly or minutes) basis. Today's situation where loads are likely to climb from the morning people get up and go to offices until noon, decline a bit while people get off work, and rise again to a point where more household appliances start being used. The phenomenon has revealed a high variation in daily power usage. In other words, peak loads are easily reached and hardly handled by the reason of similarity of every normal human being's routine, i.e., high correlation in demands at specific times.

How to cut off the power use during peak hours and intelligently swap them to off-peak periods is a challenge. Initially, one can mainly focus on controlling major household appliances or CPEs (customer premise equipments) that consume energy for an average long-term use, for example, air conditioning and heater. Other appliances or devices such as hair dryer and vacuum cleaner operating for a short-term use can all of a sudden draw a large amount of energy. This should be seriously taken into account for a more complicated and unpredictable load analysis at a later stage.

Shutting down the running appliances by utilities when the upper threshold of power usage is reached is an option to avoid over-demanding. However, consumers may be unhappy or even frustrated with such operation, e.g., hot water becomes unavailable suddenly during a shower. While the forced shutdown should only be carried out for emergency, one practical solution to effectively manipulate consumers' demands for energy use is to design an intelligent real-time pricing model. The implementation and integration of AMI, smart meters, advanced DSM, distributed energy resources/storage, and energy management units in HAN will make the operation more clever and humanistic. The energy price of TOU including peak pricing can be calculated and shown to consumers on the HAN interfaces, e.g., smart thermostats, PCs, smart appliances, and smart phones. With the useful feature and program provided, consumers will make their own decision on energy use with reference to the energy price forecast and advice. Individuals preference profiles in accordance with consumers' acceptance and routines can be built to perform "set-it-andforget-it" feature for further convenience and easiness.

While game theory can be used to describe human reaction on price change, the analytical model of a more precise

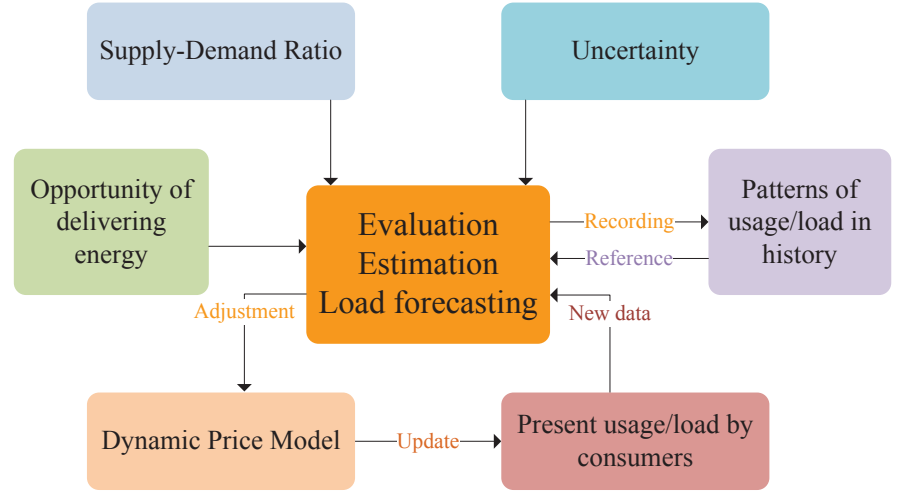

Fig. 2. The cyclic evaluation model for the process of demand response.

short-term price elasticity carried out by intelligent software multi-agents is more viable [1]. The model is essentially based on the overall ratio between supply and demand, the availability of energy, and the network capacity of delivering such requested energy. Figure 2 illustrates a refined concept on the DR process. The load forecasting is evaluated based on the acquisition of the past energy usage pattern and the contingent-but-considerable uncertainty factors. The real-time pricing is then derived via the dynamic model and becomes available to consumers. Consumers have the right to pay for the energy use at a specified time if they are willing to. The consumer usage pattern reacted to the TOU price is then fed back to the evaluation process and stored as reference for the next round pricing update. The cyclic feedback system for dynamic pricing and tariffs can help achieve smaller variations in energy consumption. By balancing the system via consumers' self-adjustment would ease the burden for utilities' load management in the long run. By means of energy shifting, the non-peak periods can be utilized for the shifted loads to avoid buying or generating more power during peak hours. At the same time, it also prevents the system and utilities from underutilizing the generated energy ${ }^{6}$.

5) To Slacken the Pace of Rising Energy Demands: Demanding more power is a serious concern akin to rising population growth. In fact, they are the cause-effect. Despite bringing the demand shifting into practice, energy demand is likely to climb sooner or later. Energy consumption is usually proportional to $\mathrm{CO}_{2}$ emission: the more energy is needed and consumed, the more energy is generated, and the more $\mathrm{CO}_{2}$ is emitted (including other deleterious pollutants). Unless power generation is no longer depending upon fossil fuels but moving to a stage of renewables-dependence in the next decades, the energy consumption issue has to be tackled. Although it might be difficult to bring down coal-based energy consumption in the short term, the following provide several possible remedies that might temporarily compensate for the forthcoming growing demand:

- Increasing the efficiency of energy use, e.g., educating consumers, implementing AMI and HAN to provide monitoring and control features, and TOU price.

\footnotetext{
${ }^{6}$ Once electricity is generated, it must be consumed almost immediately since it cannot be stored easily and possibly.
} 
- Reducing the generation and transmission losses, e.g., utilizing combined cycle for power plants generation, using advanced technologies such as Flexible Alternating Current Transmission Systems (FACTS) and High Voltage Direct Current (HVDC) for enhancing power transfer capability in high-voltage and long-distance transmission.

- Improving technologies such that appliances or electronics consume less power to operate for the same or better performance, e.g., CFL v.s. conventional light bulb.

- Extending the distributed power generating plants and renewables as well as constructing energy conserving buildings to supply the daily varying loads. It would help reduce $\mathrm{CO}_{2}$ emission and shorten power transmission distance. The more energy can be self-provided, the less is drawn from the main grid.

6) To Build a Safe and Secure System Between Supplier and Consumer: The interpretation of security is twofold. From a communications perspective, security has been an issue since the emergence of the Internet and wireless technologies in the mid 1990s. Cybersecurity seems to be a constant tug-of-war between a cohort of sophisticated cyber-attackers and a group of highly skilled defenders. Unfortunately, the egregious problem may never be eliminated but only quantified [4]. Notwithstanding the inexorable trend, the smart grid is designed to be resistant to both passive (e.g., eavesdropping, traffic sniffing) and active (e.g., DoS/Denial-of-Service, manin-the-middle) attacks that have been studied in-depth during the past decade. As an example for the intrusive DoS attack, enlarging the bandwidth may be one way to mitigate the problem, but it is not a permanent solution. A comprehensive model and strategy to safeguard the smart grid infrastructure and its diverse applications is very much in need. Khurana et al. [45] pointed out how important the effective cybersecurity solutions are needed in terms of cryptographic key management, authentication, and encryption for SCADA and PMU. Cleveland [46] also analyzed security requirements for an AMI system, such as confidentiality, integrity, availability, and nonrepudiation. Security constraints for smart meters, customer gateways, and AMI networks were further discussed in [46].

From the power perspective on the other hand, power security can be threatened by 1) intentional cyber attacks and physical attacks, 2) unintentional human errors, selfsystem malfunction caused by bugs, and severe weather, and 3) energy dependence (presented in Figure 3). For the first two cases, both cyber and physical attacks can paralyze any components in an electric power system and cause further damages to its surrounding environment, especially the most likely targeted power plants and power transmission lines. Human unawareness and carelessness, flawed software and programming codes, and nuclear power plant meltdown, can all put the power security at risk. To some degree, intentional attacks and natural disasters may not be prevented, but an effective fast-recovery procedure can be set up to restore the system within a tolerable time in order to reduce economic losses as much as possible, as well as shorten any interrupted ongoing activities or operations. On the contrary, the unintentional man-made glitches are controllable events, and hence can be averted and improved. In the case of energy

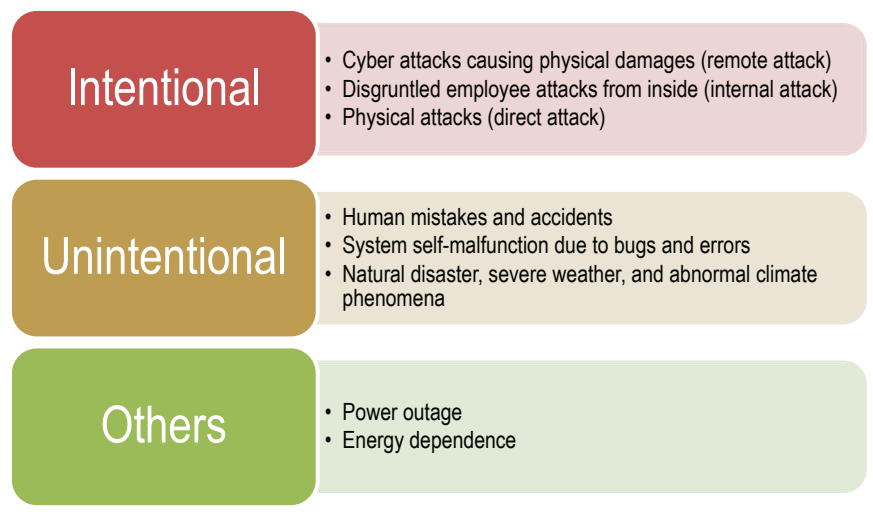

Fig. 3. Potential security threats against the electric power system.

dependence, too much reliance on the source of imported energy will also put a nation's electric power system in danger. From the study, United States, Japan, China, Germany, and South Korea were the top five worldwide energy importers in 2008 [9]. The smart grid attempts to cut down dependence on imported energy and the overall demand through energy conservation measures. Increasing the number of different energy suppliers as well as exploiting fossil fuels and RES will also promise long-term security to the nations.

7) To Reduce Unnecessary Expenditures and Recurring Costs: Costs involved in an electric power system can be categorized into capital, fuel, operation and maintenance, and power outage. Ironically, cost on investing in building the smart grid has been a top concern and barrier for the investors. It is reasonable that it may be uncertain and uneasy to foresee how much benefits the smart grid in a large scale would bring to the society at this point. In the same manner, return on invested capital may not be achieved from a short-term investment. However, the following are certain:

- Fossil-fuels consumed in power plants are becoming more expensive.

- More power plants (especially ones that burn fossil fuels) and transmission lines are required to meet the climbing demand. Moreover, their implementation can be timeconsuming.

- Operation and maintenance costs for the aging infrastructure are rising.

- Power outages incur dramatic costs to the economy and environment.

These unavoidable costs are presumably unwise, demotivating, and temporary palliative. Therefore, modernizing the power grid is imminent.

\section{Drivers for The Smart Grid}

1) Key Players: The development of the giant smart electric power system requires contributions and collaborations from governments, private organizations, and different groups of people. Since the smart grid is considered as a part of the protected critical infrastructure [47] under the Department of Defense and the Department of Homeland Security, the government is responsible for creating national strategies to deal with threats against infrastructure. Meanwhile, other players include 


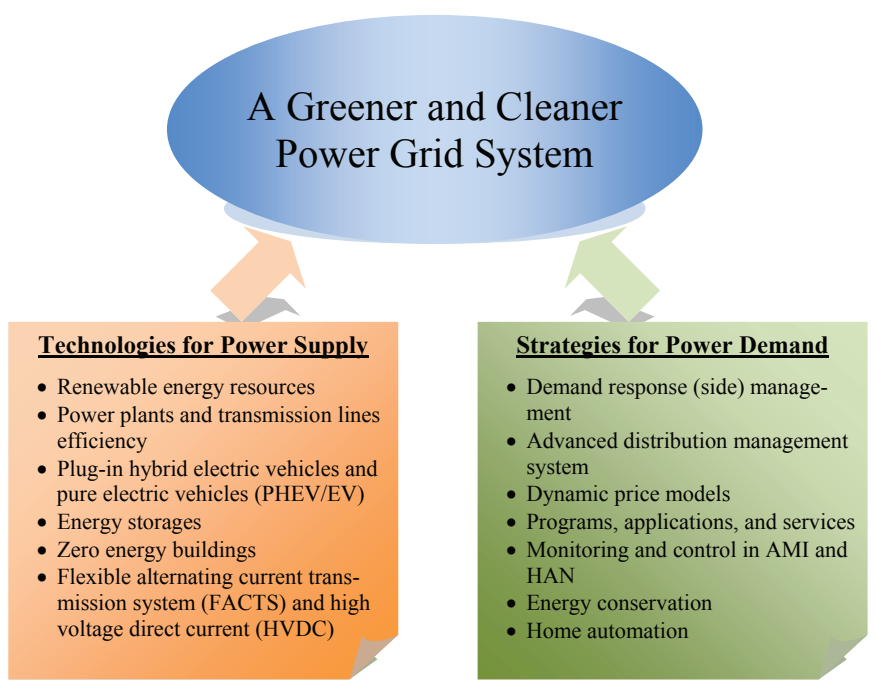

Fig. 4. Two perspectives on the achievement of the Smart Grid.

- The Federal Energy Regulatory Commission (FERC), North American Electric Reliability Council (NAERC), Regional Transmission Operators (RTO), Independent System Operators (ISO).

- Department of Energy (DOE): the National Energy Technology Laboratory (NETL), National Renewable Energy Laboratory (NREL), and U.S. Energy Information Administration (EIA).

- National Rural Electric Cooperative Association (NRECA), Edison Electric Institute (EEI), American Public Power Association (APPA).

- National Institute of Standards and Technology (NIST), Electric Power Research Institute (EPRI), and U.S. Environmental Protection Agency (EPA).

- National Association of Regulatory Utility Commissioners (NARUC), North American Energy Standards Board (NAESB).

- State assistance, e.g., regulators, policy makers, consumer advocates.

- Utility companies and stakeholders, load-serving entities, other DR providers, renewable resource developers, vendors, wholesalers, sponsors and affiliates, associations and foundations, product and service providers.

- Large commercial and industrial customers implementing DR for widespread benefits and wholesale markets, as well as residential customers for business and benefit purposes on supporting DR.

2) Key Technologies, Agents, and Strategies: Transforming the legacy electric power system into a greener and more efficient smart grid requires technologies of power engineering to meet energy demand as well as the auxiliary strategies to control demand, as shown in Figure 4. The two differentiated categories can be seen as major hardware and software facilities. The technologies category (left in Figure 4) is presented in the following:

- Renewables especially solar and wind have been mostly focused, studied, and deployed. They are more capable of generating considerable amount of energy when the requirements are achieved, i.e., available sunlight and blowing wind, as compared to others.

- Technologies assisting in lowering power loss during power conversion at the source, as well as power delivery in transmission lines, enhance the efficiency of power generation, transmission, and distribution.

- $\mathrm{PHEV} / \mathrm{EV}$ is the next generation electric vehicle which no longer merely relies on fossil fuel but electricity stored in batteries. More attractively, it is capable of providing energy back to the grid, known as vehicle-to-grid (V2G), representing the mobile electricity storage.

- Energy storage has enormous potential for the forthcoming energy revolution. They will play the roles of flexible objects stored in the future $\mathrm{PHEV} / \mathrm{EV}$, alternative sources in distribution sectors, and adaptive backup energy for immediate emergency use and relief of local demands. The system can be easily and cheaply balanced by utilizing large batteries instead of often turning power plants on and off.

- Zero energy buildings can produce energy on its own and support itself via on-site renewable resources. Hence, it does not emit greenhouse gases. Furthermore, it is able to supply its spare clean energy back to the grid.

- FACTS and HVDC are one hybrid AC-DC solution designed to improve controllability and avoid transmission congestion in the electric power system. They may also increase the distance and capability of power transmission, reduce line losses, as well as prevent cascading disturbances, for example, with the superconducting transmission cables to some extent [10].

Note that the technologies category concentrates on the effectiveness and efficiency of power supply. It ensures that electric power is generated productively and cleanly, available for delivery, delivered in bulk, and reaching anyone and anywhere whenever it is needed. Thus, power loss can be managed efficiently as much as possible. However, if consumers demand more power, the seriousness of this category escalates because of the unstoppable demand. Therefore, if a bundle of strategies and incentives for regulating the power demand exist, the unpredictable loads can be controlled effectively.

The second category strategies (right in Figure 4) introduces the next-generation solutions to assist and sustain the operation of the legacy electric power system. Such solutions will be utilizing AMI and energy conservation to develop effective and efficient demand side management as well as dynamic price models via smart meters. Programs, services, and incentives will be provided to consumers through HAN. In addition, the agent-based technologies enact considerable roles, which can be IEDs, software programs, and computerized systems (e.g., SCADA/EMS, smart appliances) employed in control and management units, e.g., operations in microgrids (MG). Multiagents must have the capability to perform data information exchange and make timely decisions in cooperation with one another. Both categories will affect each other exchangeably and promisingly provide a long-term efficient electric power system. 


\section{Projects in Progress and Survey Results}

1) Projects: A number of major countries and regions across the world have already been undertaking various smart grid projects. Below are a few examples.

- An informational list of AMR/AMI/smart grid projects and trials/pilots that the utilities in the United States have initiated [21], such as Southern California Edison [40], Xcel Energy's smart grid city, city of Boulder in Colorado [48], Austin Energy of Austin in Texas, as well as Grid Smart Ohio project from American Electric Power (AEP) and Energy Smart Miami initiative by Florida Power \& Light (FPL).

- DOE's integration-in-practice GridWise demonstration project on Washington's Olympic Peninsula [10], where consumers approximately saved $10 \%$ on their bills and peak load was reduced by $15 \%$.

- FERC's released draft for Comment of the National Action Plan on DR [49] that aims to expand DR resources, develop customer programs, and identify models and analytical tools for those who are currently involved in smart grid projects and programs.

- EPRI's IntelliGrid [50] representing the technical accomplishment and implementation projects for smart power delivery.

- GridWise Alliance's handbook [51] designed for those who are motivated to develop or access high quality smart grid projects.

- Blessing's perspective [52] on the business decision criteria considered for those who are evaluating the future choices of smart grid networking.

- Japan's project [53] on reviewing ICT infrastructure for Japan's future autonomous power system and integration of distributed PV renewable energy by CRIEPI.

- Korea's Power IT project by KEPCO [54] on development of AMI and distribution automation system.

- China's development of the AMR system using power line communication (PLC) and broadband over powerline (BPL) toward smart grid, and discussion on unity of the ultra-high-voltage power grid and smart grid [55], [56].

- Spain's GAD Project [57] on designing a communications system over distribution networks.

- Europe's ADDRESS Project [58] on the development of active demand, as well as EDP's InovGrid project in Iberia [59].

2) Surveys: According to SmartGrid News [2] in regard to Utility Survey: Pacific Crest Mosaic Smart Grid, the following show a number of raised issues on how industry leaders think of budgeting and selecting technologies for smart grid projects.

- Cybersecurity has been selected as the top one standard followed by the smart meter, HAN, wide area network (WAN), auto DR, and border interface standards.

- Communications is the most important item as compared to meters, substation automation, outage management, DR, advanced control system, voltage monitoring, and HAN integration.

- Mesh network is considered to be the most important communications technology for the smart grid as compared to point-to-point Radio Frequency (RF), Worldwide
Interoperability for Microwave Access (WiMAX ${ }^{\mathrm{TM}}$ ), cellular, BPL, paging and public wireless networks, Wireless Fidelity $\left(\mathrm{Wi}-\mathrm{Fi}^{\mathrm{TM}}\right)$, and satellite.

- Advanced control is the most crucial long-term application with respect to $D R$.

- Cost is the strongest barrier to smart grid projects, notably followed by technology immaturity.

- Reliability improvements is the top driver of expenditures for smart grid projects with $D R$ and peak shaving placed right behind.

- Distribution automation has been polled as the top smart grid application.

- Systems integration, data management solutions and electric vehicle integration are the primary deployment concerns.

From the 2010 North American Utility Smart Grid Deployment Survey taken by smart gird industry leaders from over 50 North American utilities [60], over $60 \%$ of respondents claimed themselves to be in the deployment stage with a formal strategy in place while other $31 \%$ were still in planning and investigatory phases.

In addition to the conducted surveys, ten sagacious audit questions presented in [6] provide a tour guide for electric utilities to review and evaluate during the smart grid development stage.

- Having at least one senior professional in an organization.

- The capability of doing business with customers.

- Being clear to customers about what services are provided and how much they cost in order for customers to make timely and consistent decision to manage their consumption and costs.

- Providing monitoring and control technologies as well as features to customers.

- The effectiveness of having and using an accurate and detailed circuit model of distribution systems all the way to the end users.

- The necessity of having an automated outage management system that incorporates the detailed circuit model.

- The need for geographic information systems that subsumes the connectivity and analysis of the detailed circuit model.

- The deployment of SCADA throughout the distribution grid.

- Having strategies and plans of taking maximum advantage of the Internet.

- Contingency plans for an extended power system outage. The number of smart grid players and relative projects is growing. A few more examples include the recent $\mathrm{Cisco}^{\mathrm{TM}}$,s Smart Grid Strategy from good and bad aspects, Silver Spring ${ }^{\mathrm{TM}}$ 's Smart Energy Platform, $\mathrm{GE}^{\mathrm{TM}}$,s Smart Grid Problem on home energy management and PHEV charging, and IBM $^{\mathrm{TM}}$ 's Smart Grid Strategy about being a super integrator [2].

A sea of long-term solutions rather than short-term palliatives are much desired. The groundbreaking smart grid modernism in the so-called Energy-Climate $\mathrm{Era}^{7}$ [5] in the

\footnotetext{
${ }^{7}$ December 31, 1999 was the end of a century (or of a common era). Meanwhile, January 1, 2000 was the first day of the first year of a new era, namely, the Energy-Climate Era (1 E.C.E.).
} 


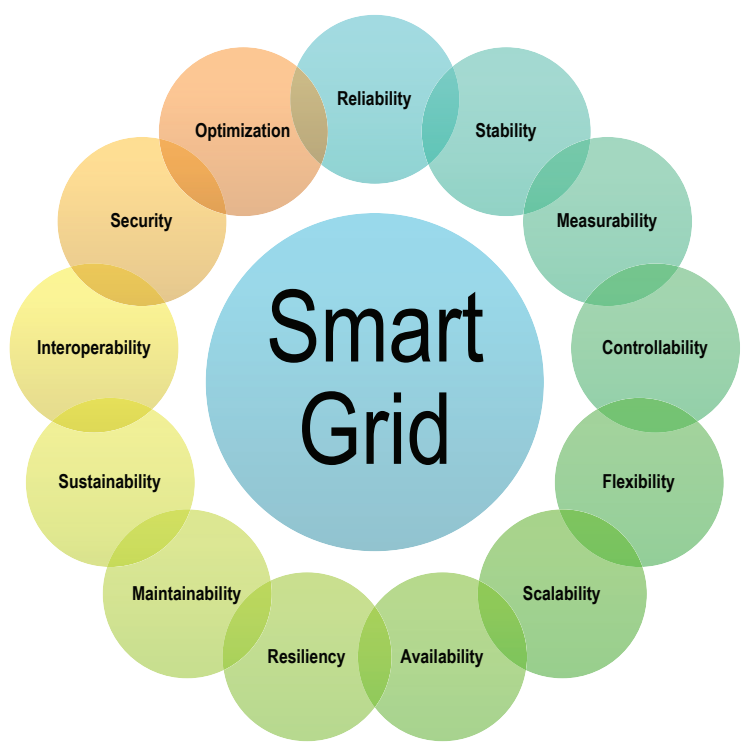

Fig. 5. The Smart Grid characteristics and requirements.

Twenty-First century will show the importance of innovation, collaboration, and leadership throughout nations and the globe. The smart grid integrated with the current state-of-theart technologies in power, communications, and information, is envisaged to bring a more intelligent and environmentally sensitive network to the existing system. Furthermore, it will 1) initiate the untrammeled markets and economic growth, 2) create job opportunities, 3) provide effective grid asset and cost management processes, 4) explore diverse innovations, and 5) draw a wide range of research interests, for the nextgeneration electricity economy and digital world.

\section{Challenges to The Quality of Power And COMMUNICATIONS FOR THE SMART GRID}

The following elaborate on a series of essential attributes for the smart grid, as shown in Figure 5. As a matter of fact, they are interconnected in a very close relationship as cause-effect among one another. Each of them should be well considered for the progressive strategies and designs in both power and communications fields to support the smart grid development.

\section{A. Reliability and Stability}

Reliability has a durability feature. In general, it interprets the operational health and degree of volatility of the entire system. It further presents the state of high consistency, repeatability, and dependability that the smart grid will maintain in accordance with effective measurements and assessments. The stability of a system determines the level of reliability for which the system is qualified. Meanwhile, reliability is dependent upon the achievement of other contributing and decisive factors, described in the following subsections. The smart grid must guarantee voltage and current stability, mitigate peak demand and load variability with implementation of DG and energy storage over wide areas, and preclude a variety of incidents.

\section{B. Measurability and Controllability}

Service interruption and faults are serious and possible to happen. It is important for them to be measurable and controllable in such a way that deliberate evaluations and assessments can take place. The smart grid is capable of identifying and correcting disruptive operations through dynamic measurements and control monitoring in real time. In the meantime, some degrees of observability and transparency should be obtained in order to efficiently analyze, manage, as well as predict and respond to varying network conditions. A wealth of data information, which considerably makes the grid smart, must also be measurable, observable, and manageable.

\section{Flexibility and Scalability}

The grid is moving from a centralized infrastructure to multiple decentralized MGs. Scalability of the electric power system has to be well determined. By means of islanding, MGs attempt to incorporate DG and energy storage for contributing energy to utilities as well as to local served areas during times of peak demand. The island functionality [61] introduces a concept of a giant smart grid composed of multiple micro smart grids. Each local grid is able to operate autonomously with respect to DSM, quality and reliability model, fault management, and security management. While fulfilling the scalability goal, flexibility allows the smart grid to provide multiple redundant alternate routes for power and data to flow, as well as supply options for feasible control and operation when needed. Flexibility may also apply to a set of standards exercised in the smart grid such that they should be available and upgradeable throughout the world including ANSI, IEC, PLC, wireless M-Bus, and ZigBee.

\section{Availability}

The availability of power and communications is essential upon consumers' requests for energy and information. It relies upon the availability of data exchanged in the network. A high degree of resource availability is required especially when dealing with the latency and security issues. For examples, a latency of few tens of milliseconds should be achieved in line protection and control systems; a DoS attack can aggravate network performance causing servers or services temporarily unavailable. The redundancy measure might be a foreseeable resolution to the problem. However, its effectiveness will depend upon how the system should be designed while avoiding the consequent costs of high network complexity as well as the scalability matter.

\section{E. Resiliency}

The degree of resiliency determines how reliable the smart grid actually is when incidents happen. Especially from the safety and security perspectives, resiliency represents the capability to restore and recover from various disruptions or malfunctions through the robust fast-response process. The vulnerable electric components in the grid are likely transmission lines and stations, large power plants, and nuclear plants with leakage. Multiple contingency plans are required to address any failures or destruction caused by natural disasters, deliberate attacks, or malicious activities. 


\section{F. Maintainability}

Maintainability fundamentally reflects longevity and reliability of a system. It usually shows the ability to effectively and efficiently perform a series of actions for maintenance tasks. Procedures specifically executed in maintenance involve inspection, troubleshooting, and replacement. The smart grid must be designed for ease of maintenance such that the diverse power and communications components (e.g., facilities, equipments, systems, subsystems, network security and management) would be repaired rapidly and cost-effectively. Likewise, high efficiency in manhour as well as tools and equipments plays a critical factor for the maintenance system of the grid network.

\section{G. Sustainability}

The rise of environmental concerns and peak demand risks has made sustainability a crucial requirement for the grid operation. A sustainable power ecosystem promises to provide sufficient greener energy with the interactive supports and contributions from local MG as well as the main grid. It also helps facilitate efficiency to optimize the system balance and grid assets. In the meantime, the environmental-friendly and user-friendly attributes will motivate the development of RES over wide areas as well as customization through the customers engagement.

\section{H. Interoperability}

The efficiency and effectiveness of the overall system performance will dominantly depend upon the completeness of interoperability engaged in the infrastructure. Elements of constructing the smart grid entail a number of standards being interoperable and common for interconnection of power as well as communications technologies. Such capability is needed while integrating and converging varieties of technologies and communications protocols in order to understand each other and offer seamless power and data transport. Clumsy interaction and integration among diversities would slacken the response time as well as degrade the overall system operation and throughput.

\section{Security}

Bridging a safe and secure connectivity between suppliers and consumers provides protection for critical applications and data as well as defenses against security breaches. Several existing security measures and tools are elementary requirements in the smart grid security, such as Firewall, intrusion detection systems and intrusion prevention systems (IDS/IPS), virtual private network (VPN), virtual local area network (VLAN), and Access Control.

While most of the security issues have been discussed in Section II-B6, the inclusion of customers participation initiated by the smart grid plan is likely to induce customers' privacy concern [18], [21], [45]. Energy usage patterns generated by household appliances may be revealed to the intended

\footnotetext{
${ }^{8}$ Popular protocols used in VLAN networks among organizations and enterprises are defined in standards, e.g., IEEE 802.1D-2004 RSTP and its extension IEEE 802.1Q-2005 MSTP.
}

intruders during data transmission. Such migration of data communications and exchange into premises would be challenging. How to earn consumers' trust is one of the nontrivialities to success. In addition to aforementioned active and passive intrusions as well as the gravity of data confidentiality and integrity, the emerging smart grid has to address the following issues:

- What information should be contained in data? Should every single datum be collected and delivered to utilities to support load management as well as monitoring and control?

- How often should data be transmitted?

- How would data be administered and stored for future analysis? Who would be in charge of data management and data storage? How long should data be kept?

- What solid security mechanisms should be adequately employed for the typical smart grid security?

Innovations in data security as well as privacy for the smart grid are much desired.

\section{J. Optimization}

Optimizing the smart grid operation and assets is imperative. Optimization can be facilitated with the support of the advanced technologies and IEDs as well as intelligent automation and management, while balancing a diversity of variables and tradeoffs. The smart grid is to be optimized in terms of 1) reliability of power delivery, 2) efficiency of power conversion and use, 3) quality of power generation and distribution, 4) availability of power and data transport, 5) effectiveness and accuracy of data and communications, 6) fault management and time response, and 7) economic profit. In the meantime, reducing the capital cost, network complexity, and recourses usage, is crucial for the smart grid to be practically developed.

\section{K. Mobility}

An enormous amount of smart meters will be installed worldwide in the forthcoming years. Locations where smart meters being deployed outside single-family houses or inside designated rooms in condominiums would affect the performance of RF transmission as well as the effectiveness of cooperation between PLC and RF communications in AMI and HAN. Since their positions will be fixed and static, smart meters should be strategically placed to avoid unwanted location-dependent limitations (e.g., interference) as much as possible. While line-of-sight (LOS) may not be possible especially in urban areas, at least no major non-LOS (NLOS) impediment or obstructions (e.g., buildings, trees, parked vehicles) should be present to interrupt signals among smart meters as well as the corresponding endpoint receivers.

\section{Power Level and Receiver Sensitivity}

In addition to the issue of NLOS signal transmission, the design of power level and receiver sensitivity for the smart meters should also be appropriately determined. Lowering the power level might be preferable to environments affected by NLOS impediment. Likewise, increasing the receiver sensitivity would allow smart meters to differentiate unwanted noise 
and other disturbance signals from the captured signals more easily. How to select adequate values for these variables while balancing the tradeoffs is a critical subject. They are likely to affect the overall performance of entire smart metering network.

\section{Energy Consumption}

Energy consumption in wireless networks, especially in wireless sensor networks (WSN), has always been the top priority over other network performance metrics. In other words, the constraint of energy-efficiency has always been taken into consideration in designing algorithms and mechanisms for WSN. Major sources for energy wastage include collision, idle listening, control overhead, and overhearing. Fortunately, most devices such as smart meters, thermostats, water heater, and household appliances, are wire-powered in AMI and HAN, and therefore powering is no longer an issue. However, more power-efficient schemes are still desired, especially for battery-powered mobile handheld electronic devices, remote control, and monitoring sensors, in order to save as much energy as possible, i.e., to prolong the longevity of these devices.

\section{N. Data Traffic and Prioritization/Classification}

In the smart grid scenario, the majority of data is somewhat deterministic since, by default, smart meters may periodically send information back to the utilities every 60 minutes for residential energy use, 15 minutes for commercial use, and 24 hours for gas and water meters data retrieval. While the time period of data collection from various sources for different purposes can always be adjusted based on utilities' needs, the overall traffic pattern can be seen as ON/OFF and bursty. In order to effectively manage mountains of data generated in the smart grid, mechanisms for the network resource allocation and scheduling should be strategically planned and designed.

How data packets should be handled and transported is a major topic. Essentially, one can categorize and prioritize data so that these data will be treated appropriately during data transport through various paths/routes and possibly across heterogeneous networks before reaching the destination. The alternative is to establish dedicated channels and routes by using private facilities for communications. More importantly, how utilities can offer consistent scrupulous surveillance and regulations to secure their communications networks and data is not an easy task.

In addition to the periodic data collected for load management and maintenance purposes at utility centers as mentioned earlier, it is necessary to classify data into different groups. Data are treated to be time-critical based on the occurrences of abrupt emergency, disaster, and the corresponding consequences. Examples include voltage drops, switching commands, and physical network links removals for maintenance during normal operation or abnormal destruction. The data should be distinguishable, handled differently in terms of class of service, and not interspersed with others, e.g., the general data from the Internet, voice data from phone calls, video data streaming from particular public safety communications, as well as traffic signal controls. Once data transport is carried

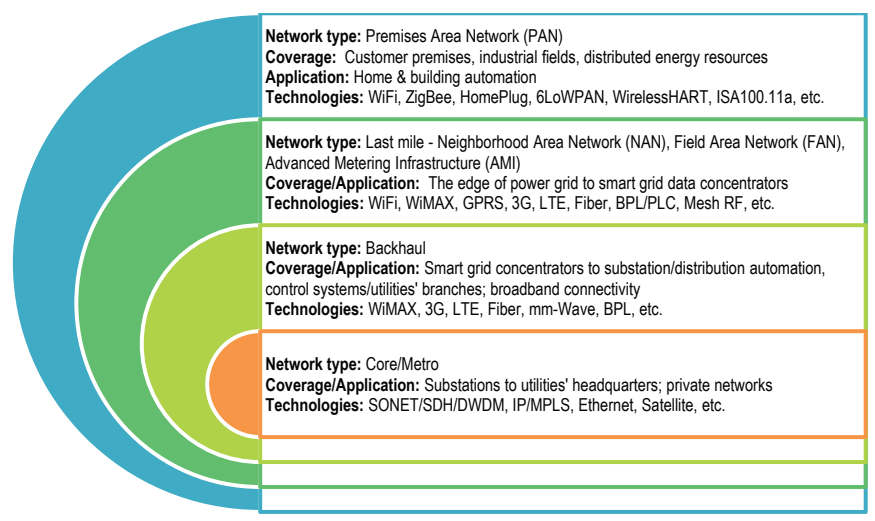

Fig. 6. The Smart Grid communications layer.

out in both uplink and downlink ${ }^{9}$ directions, any suspicious data traffic patterns must be well supervised and assessed.

\section{From Communications Perspective: INTEGRATION OF TECHNOLOGIES}

The smart grid is involved with data sensing, collection, transmission, communication, and storing. A vast amount of sensor-based devices and LEDs are deployed in electric components as well as homes and premises. The collected data from these devices are aggregated and delivered to control centers for operators or automated systems to measure, analyze, and execute the process of planning-prediction-prevention. The successive operations help facilitate monitoring and control to increase the workload efficiency and strengthen the security protection of the grid. The two-way and end-toend communications feedback network system will require a robust and elaborate infrastructure to support itself. Likewise, a sturdy WAN supporting power system operations is much desired to handle data transport under various smart grid machine-to-machine (M2M) applications [62].

The smart grid communications infrastructure is essentially composed of four networking sectors: core (or backbone, metro), middle-mile (or backhaul), last-mile (or access, distribution), as well as homes and premises. Figure 6 illustrates the typical communications layer for the smart grid. The core network supports the connection between numerous substations and utilities' headquarters. The WAN requires high capacity and bandwidth availability to handle mountains of data transported from other sectors as well as multiple agents. The backbone network is usually built on optical fibers.

The middle-mile connects the data concentrators (or aggregators) in AMI with substation/distribution automation and control centers associated with utilities' operation. This sector not only needs to provide broadband media, but requires its network installation to be as easy and cost-effective as possible. In addition, routes and links through which data flow ought to be flexible and uninterrupted. More importantly, the overall performance is predictable for reliable data transport before entering the core.

\footnotetext{
${ }^{9}$ Uplink: from devices/child nodes to their associated network coordinators, SCADA/PMU sensors to control centers, smart meters to utilities, and vice versa for downlink.
} 
The last-mile covers the areas of FAN/NAN and AMI; it is responsible for both the data transport and collection from smart meters to concentrators. There are a variety of wired and wireless technologies available to be implemented in this sector. The tailored technology must provision broadband speed and security.

The premises network is gaining more attention for the last sector of the smart grid. Communications technologies supporting HAN as well as associated building automation will be predominantly based on the IEEE 802.15.4, IEEE 802.11, and PLC standards. Home energy management operated in HAN will regulate numerous components, such as thermostat, HVAC, smart appliances, lighting control, home automation, $\mathrm{PHEV} / \mathrm{EV}$, and DG. Data collection and transport from this sector have to be stabilized, accurate, and secured.

The four networking sectors interconnecting with one another supported by various technologies fundamentally assemble the communications infrastructure of the smart grid. The following subsections will introduce the dominant technologies and discuss the integration challenges for the smart grid infrastructural development and implementation.

\section{A. Technology Candidates for Supporting Communications in Smart Grid}

Popular physical media used in signals transmission are copper, fiber, and radiowave/microwave. Copper has the longest history among the three. Fiber, which is immune from electromagnetic interference, can provision high bandwidths over longer distances, and is a better alternative.

Contrary to wireline, wireless technology is viable to solve communications gap [63]. Additionally, radiowave possesses cost effectiveness on capital and recurring expenses as well as fast deployment. However, radiowave may achieve lower throughput as constrained to wireless impairments and interference, dead spots (e.g., 2G/3G), and shorter-distance coverage, e.g., Wi-Fi. Adoption of technologies will likely be based on the business decision, the geographical service needs, and the type of networks for the backbone and spur segments of the smart grid infrastructure.

1) Wireline (or Wired) Technologies: Wireline technologies such as fiber and BPL may be preferable options for utilities when they are available already in the served areas and their performance requirements can be achieved. Fiber-optic and optical network technologies, such as the next-generation Synchronous Optical Networking and Synchronous Digital Hierarchy (SONET/SDH), are capable of offering different data rates at the access, aggregation, and core levels. They provide multi-service provisioning platforms that support IP and Ethernet applications [64]. As a result of Ethernet's simplicity and cost-effectiveness, adopting IP with MultiProtocol Label Switching (MPLS) to achieve Ethernet transport over SONET/SDH on its existing packet switched networks (known as carrier Ethernet) will enhance reliability, quality of service (QoS), and security for the critical applications of the smart grid. Ethernet is currently able to provide speeds of $1 \mathrm{Gbps}$ in Gigabit Ethernet (GbE) and $10 \mathrm{Gbps}$ in 10GbE. The emerging $40 \mathrm{GbE} / 100 \mathrm{GbE}$ with abundant capacity will be beneficial to the aggregate smart grid data traffic.
Likewise, Ethernet and Gigabit passive optical networks (EPON/GPON) [65], [66] use diverse optical-electrical approaches to provide sufficient capacity for large data delivery as well as high speed data transmission in access networks. They take advantage of Wavelength Division Multiplexing (WDM) and deploy fiber to the end-user (FTTx) to serve groups of premises and neighborhoods. Utilizing different wavelengths for both upstream and downstream traffic simultaneously enables high flexibility in routing and switching optical signals.

On the other hand, PLC has existed for quite some time in electric power systems. The purpose of PLC was to use power line as the communications medium to provide a communications network such as the Internet while supporting the conventional services related to power distribution, e.g., load control, and (remote) metering reading. PLC fundamentally comprises the MV transmission network as well as the LV distribution network. Technologies used in PLC are mainly narrowband (NB) operating at low frequencies (several $\mathrm{kHz}$ ) and broadband (BB) operating at high frequencies (hundreds of $\mathrm{MHz}$ ). As comparable with IEEE 802.3 Ethernet, the medium access protocols used in a PLC infrastructure are also based on topologies of the master-slave-oriented star polling, ring token passing, bus with reserved Time-Division Multiplexing (TDM), and the random Aloha or Carrier Sense Multiple Access with Collision Detection (CSMA-CD) technique [14], [67]. The main advantages of implementing PLC include 1) inexpensive rewiring of buildings by using the existing wiring, and 2) power-supplied devices on premises being able to communicate in the network once plugged into the AC outlets. NB-PLC is essentially employed in MV and LV networks whereas BB-PLC (i.e., BPL) provides broadband Internet access via a BPL modem that is connected to any outlet in PLC-networked homes and buildings. A newly discovered mechanism called E-Line is able to support a much higher data rate in excess of $1 \mathrm{Gbps}$ and operate in bands between $100 \mathrm{MHz}$ and $10 \mathrm{GHz}$ [68].

Unfortunately, PLC also struggles with the problems of attenuation, noise, and distortion found in RF communications when communicating over the electrical power wiring. Since power line was not initially designed for data transmission, a number of critical issues and challenges need to be tackled in PLC:

- Varying impedance and channel condition;

- Non-white-noise in nature;

- Frequency-dependent attenuation associated with outlet locations, geographically different wiring structures, and the number/type of connected electronic devices being turned $O N$ over time while plugged in to power lines;

- Phase switch/change (from single-phase to three-phase and vice versa) between indoor and outdoor architectures.

There have been studies in PLC research and deployment. For instance, Sarafi et al. [69] discussed the actual BPL deployment project in central Greece that used a hybrid wireless-BPL solution for rural areas. The goal was to combine BPL and Wi-Fi as the last mile access with QoS assurance provided for its data transmission through the MV power grid.

Bumiller et al. [67] introduced the concept of a singlefrequency network that allows all repeater nodes in multi-hop 
transmission to relay data packets simultaneously operating in the same frequency band. Interestingly, a flooding mechanism was applied to eliminate most of routing overhead requirements for the PLC network. Furthermore, Bannister et al. [70] proposed to employ Orthogonal Frequency-Division Multiple Access (OFDMA) in PLC by combining frequency diversity and orthogonality to adduce PLC-specific issues, as opposed to the current single carrier systems with Frequency-Shift Keying (FSK) and Binary Phase-Shift Keying (BPSK), as well as the potential candidate Code Division Multiple Access (CDMA), which may be less robust on its carrier frequency selectivity. Various standards for PLC including IEEE P1xxx (P1675, P1775, P1901), OPERA, POWERNET, UPA, CEPCA, ETSI PLT, as well as HomePlug series in 1.0, AV, BPL, and CC specified by HomePlug ${ }^{\mathrm{TM}}$ Powerline Alliance, can be further studied in [71] and [72].

2) Wireless Technologies: Telecommunications technologies such as mobile/cellular networks and wireless LAN WiFi networks are quite matured and readily deployed today. Cellular mobile networks are mainly based on $2 \mathrm{G}$ and $3 \mathrm{G}$ technologies: Global System for Mobile communications (GSM), Interim Standard 95 (IS-95) or cdmaOne, CDMA2000, and Universal Mobile Telecommunications System (UMTS).

GSM is a $2 \mathrm{G}$ cellular technology using Time Division Multiple Access (TDMA) channel access method. Its backwardcompatible newer version, for example, General Packet Radio Service (GPRS) provides packet data capabilities to the original GSM system, and Enhanced Data rates for GSM Evolution (EDGE), standardized by Third Generation Partnership Project (3GPP), is considered as a $2.5 \mathrm{G}$ transitional technology capable of higher data rate, IP traffic, and text messaging. GSM band carriers include AT\& $\mathrm{T}^{\mathrm{TM}}$ Mobility LLC (with Cingular $^{\mathrm{TM}}$ merged in 2005) and T-Mobile ${ }^{\mathrm{TM}}$ USA. T-Mobile is potentially to be fully merged with AT\&T in 2011-2012, thus making AT\&T the GSM monopoly in the U.S.

IS-95 is a $2 \mathrm{G}$ mobile telecommunications standard that firstly uses the CDMA scheme. Its successor CDMA2000 or IS-2000, an early 3G technology, which has evolved into CDMA2000 EVolution-Data Optimized (1xEV-DO), provides high packet data rate in air comparable to wired broadband. EV-DO (including its Revision A and B), standardized by 3GPP2, applies both CDMA and TDMA multiplexing techniques to enhance the throughput of each individual user as well as that of the overall system. Verizon ${ }^{\mathrm{TM}}$ Wireless (with Alltel Corp. merged in 2009) and Sprint ${ }^{\mathrm{TM}}$ Nextel Corp. are CDMA carriers and both of them use EV-DO for their $3 \mathrm{G}$ cellular networks.

Another CDMA-based 3G technology, UMTS, also known as Wideband CDMA (WCDMA), represents an evolution of GSM sometimes named 3GSM. It applies International Mobile Telecommunications - Direct Spread (IMT-DS) using spreading bandwidth of $5 \mathrm{MHz}$ [73] to provide high speed data, better quality of voice, and the ability to access the Internet and packet applications. The $3 \mathrm{G}$ transitional protocol standards such as High Speed Packet Access (HSPA) for both uplink and downlink, and later the Evolved HSPA (HSPA+) are currently available to improve the performance of the existing WCDMA. AT\&T and T-Mobile fall into this category. The pre-4G technology 3GPP Long Term Evolution (LTE) and the intended 4G LTE Advanced designed to increase the capacity and speed of its cellular networks have been more attractive to the adoption of carriers such as AT\&T, T-Mobile, and Verizon. The most attractive advantage of adopting LTE Advanced to be the $4 \mathrm{G}$ network for the smart grid is that the cost of upgrading on the existing $3 \mathrm{G}$ network for those incumbent carriers and hardware companies can be minimized.

The other wireless technology such as Wi-Fi, certified by the Wi-Fi Alliance, is based on the IEEE 802.11 WLAN standard. It defines IEEE $802.11 \mathrm{a} / \mathrm{b} / \mathrm{g} / \mathrm{n}$ and operates in the 2.4 and $5 \mathrm{GHz}$ frequency bands. Wi-Fi serving as the lastmile access on premises is ideal for Voice over IP (VoIP) applications. It offers net data rates between $1 \mathrm{Mbps}$ and 54 Mbps for IEEE $802.11 \mathrm{a} / \mathrm{b} / \mathrm{g}$ that operates in a $20 \mathrm{MHz}$ channel [74]. IEEE 802.11n, an amendment to IEEE 802.11-2007, is Multiple-Input and Multiple-Output (MIMO) four-streamsbased that supports higher net data rates in the range of 26 Mbps to $600 \mathrm{Mbps}$ operating in 20 and $40 \mathrm{MHz}$ channels [75]. A Wi-Fi network is constructed of one or more serving access point(s) with a number of served devices. For network initiation and establishment, the AP broadcasts packets containing Service Set IDentifier (SSID) in the header to the connected devices so that the corresponding network domain for each device it serves within its range can be specified. Unfortunately, Wi-Fi is prone to obstacles of missing roaming and authentication features, as well as the range it serves. Hence, the current and coming 4G technology WiMAX IEEE 802.16-2009 (known as wireless metropolitan area network or WMAN) and P802.16m/n that supports both fixed and mobile broadband wireless access is more likely to supplement WiFi to provide higher stability and reliability for the last mile connectivity. WiMAX provides different classes of services assignment and level of priorities in its $4 \mathrm{G}$ network to ensure that QoS is served for critical infrastructures, such as the smart grid. Sprint and its collaborator, Grid Net ${ }^{\mathrm{TM}}$ Inc., are two of the biggest proponents of building WiMAX to deliver smart grid solutions.

Wireless mesh network (WMN) [76] and low-power and low-rate (LPLR) network play vital parts in the smart grid communications infrastructure. WMN, initially designed for community or neighborhood communications, is considered as one of the foreseeable approaches to support the smart grid applications. WMN is mostly based on IEEE 802.11 to provision reliable and cost-effective mesh networking with easy installation and deployment as well as affordable investment. It may efficiently provision and manage smart grid applications, along with other non-smart grid uses at the same time via the super mesh routers deployed in WMN. On the other hand, the LPLR network is generally standardized under IEEE 802.15.4 composed of numerous sensor-based devices. WSN and Low Rate Wireless Personal Area Network (LRWPAN) are the two typical examples. Both of them are key contributors that conduct data collection and relay as well as provide connectivity among and beyond smart meters.

WSN has been extensively studied and gaining much attention from academia and industry in the past years [77]. Along with its attractive practicality and diverse applicability, WSN tailored in a wide diversity of applications involves military, security and surveillance, as well as monitoring and 
control in environment, industry, telemedicine/healthcare, and home/building automation. Sensors deployment tends to grow in quantity per system as well as size and variety in numbers of networks by a factor of two or three over the next five years [78]. The survey conducted in [78] further reveals that low power consumption and longevity are interchangeably important depending upon where and for what purposes WSNs are applied. Voluminous surveys and literatures on WSNs and ad-hoc networks have covered a variety of research topics. They can be instrumental resources in investigating WLAN and WSN for the smart grid. Issues include time synchronization [79], power consumption [80], medium access control (MAC) [81], [82], routing and fault-tolerance [83], [84], data aggregation and clustering [85]-[87], coverage and connectivity [88], [89], multimedia [90], [91], QoS [92], as well as security and privacy [93]-[95].

WPAN shares common features of wireless ad hoc networks including MANET (mobile ad hoc network) and WSN. The network is characterized with low complexity, low data rate, low energy consumption, and low cost. It supports short range wireless communications for different scenarios of environments, typically in industrial fields and home/building automation. Contrary to Bluetooth, ZigBee [96] is a foreseeable standardized technology for the emerging smart energy applications in terms of longer range support and larger number of devices that can be coordinated in its network. Meanwhile, other standards such as WirelessHART (i.e., an extension of the HART Protocol) and ISA100.11a, are suitable alternatives for industrial process automation and field testing [97]-[100].

3) Smart Meters: Smart meters constitute a mesh communications network essentially measuring the usage of electricity and possibly water/gas in AMI. They require complementary skills of PLC and RF technologies in both hardware and software to provide dedicated smart grid solutions. A marked contrast between Distribution Line Carrier (DLC) in PLC and meshed RF network is presented in [101]. Smart meters basically have multiple built-in interfaces supporting different wired and wireless communications protocols of PLC and RF technologies. Examples include modulations in PLC (e.g., OFDM, SFSK or spaced frequency-shift keying), RFbased Wi-Fi, HAN, ZigBee, 6LoWPAN for in-home displays or touch panels, open serial interface for home networks, and (wireless) M-bus for water and gas meters management. Smart meters run as a set of bridges between the AMI and customer gateways in HAN. Optimization in dynamic pricing, outage management, and other critical elements based on network diagnoses derived via AMI is imperative. A huge potential of interconnecting RF technologies with the existing PLC in the electric power system requires further investigation and exploration.

4) The Integrated Communications and Information Path Infrastructure: By effectively employing technologies apropos what has been discussed earlier, an integration of the heterogeneous networks will help facilitate efficient communications for the smart grid system. Figure 7 illustrates an overview of various components throughout the entire smart grid. The integrated infrastructure specifically includes:

- Home and building automation in HAN, BAN, IAN;
- AMI comprised of distribution automation, smart meters, smart appliances, PHEV, DG, FAN, NAN, etc.;

- Data concentrators collecting data from smart meters in AMI;

- FAN and NAN deployed with routers/gateways or lastmile access points owned by either utilities or third parties based on their rules and regulations for efficient data and security management before reaching WAN;

- Distributed renewable resources allocated close to the served areas;

- A variety of function domains, e.g., utilities, operation control centers, substations, enterprises, markets, service providers, and wholesalers, forming an interactive multiagents network.

Home automation and smart energy in HAN are based on IEEE 802.15.4, IEEE1394 ${ }^{10}$, HomePlug, Wi-Fi, and others [103]-[106]. A customer gateway may be needed to perform address conversion and translation of different communications protocols between the smart meter and WPAN coordinator in HAN. For instance, smart meters will presumably be assigned 128-bit IPv6 addresses while logical 16bit short or 64-bit extended addresses are allocated in HAN. The computerized automation system in HAN will not only intelligently monitor and control the home energy usage, but also consolidate the sustainable PHEV/EV and DG.

The adoption of various technologies for the smart grid communications will ultimately depend upon the associated network characteristics, as shown in Table II. Small utilities may take the advantages of using the existing cellular networks and collaborate with others to reduce capital and operating costs. On the contrary, large utilities would be more capable of building their own networks to avoid bandwidth sharing in order to earn more profits on the capital investment. In addition, the geographical needs, task objectives, as well as applications and services to consumers will also affect the choices of technologies deployment of the smart grid communications.

\section{B. Issues of Coexistence and IP Migration}

Converging different communications protocols entails correlating data from heterogeneous networks throughout the infrastructure. Several major issues are addressed below before acquiring reliable communications:

1) Coexistence: In wireless networks, the varieties of wireless technologies sharing the common $2.4 \mathrm{GHz}$ ISM frequency band may incur severe interference to one another. Some research studies have shown that IEEE 802.15.4 is likely more vulnerable to the disturbance caused by IEEE $802.11 \mathrm{~b} / \mathrm{g}$ and IEEE 802.15.1 (i.e., Bluetooth) has more impact on IEEE 802.11 in terms of packet drop [107]-[109]. Nevertheless, Pollin et al. [110] warned that the impact of IEEE 802.15.4 on IEEE $802.11 \mathrm{~b} / \mathrm{g}$ can be quite significant as well even when the technique of listen-before-send is incorporated. The main reason is that the time slot sizes used in both protocols are disparate; they are unsynchronized to each other due to the duty-cycle requirement operated in IEEE 802.15.4 and lack of

\footnotetext{
${ }^{10}$ This standard is known as FireWire ${ }^{\mathrm{TM}}$ that supports up to 3.2 Gbps data rate in contrast to USB 2.0's $480 \mathrm{Mbps}$ and 3.0's 5 Gbps [102].
} 


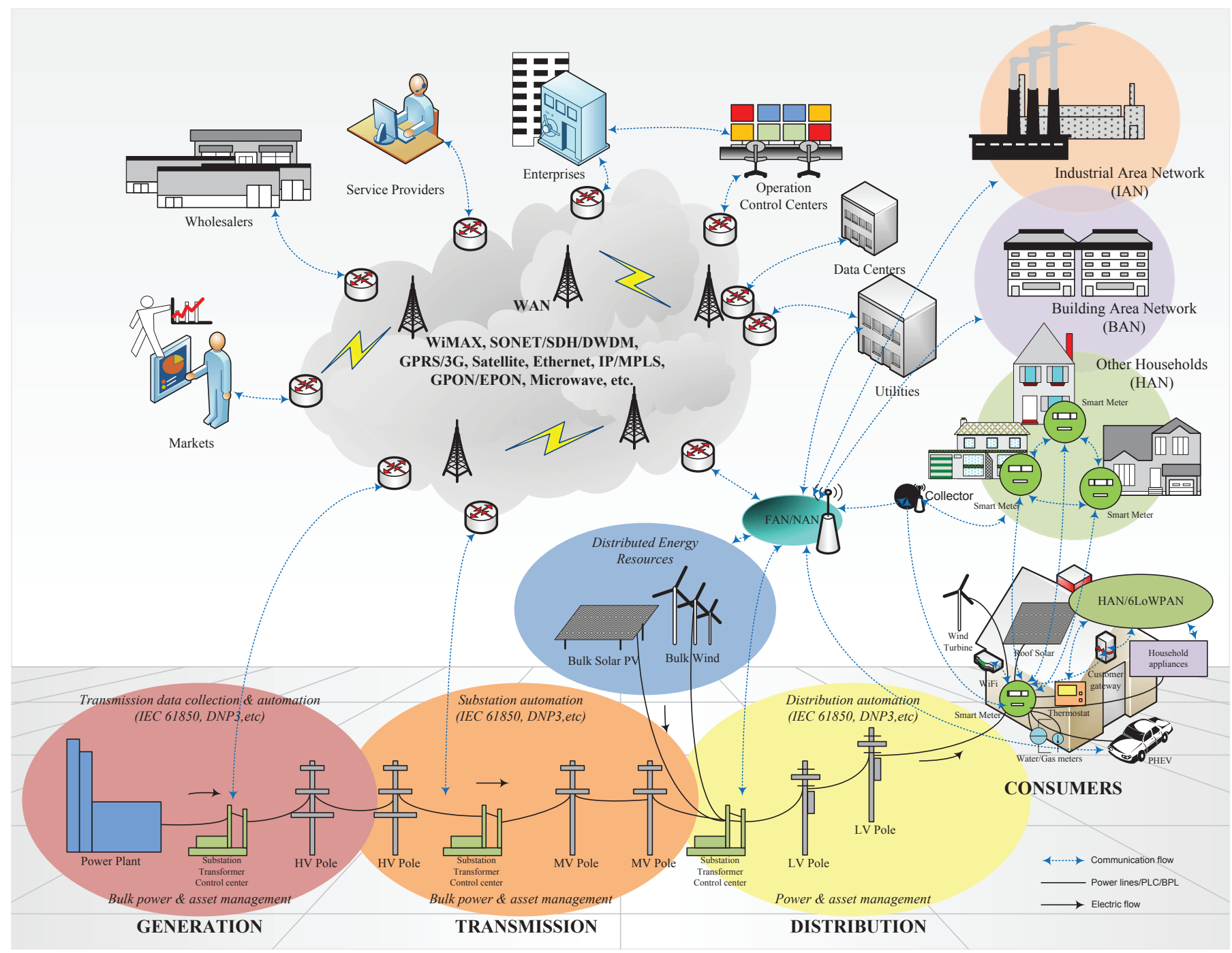

Fig. 7. The overall Smart Grid communications infrastructure.

interaction between two heterogeneous networks. Likewise, interference is also likely to occur in indoor and outdoor environments for PLC in both wired and wireless links.

The coexistence issue is critical since quite a number of wireless networks in homes are using the same frequency band for communications. The most prevalent examples are $\mathrm{Wi}-\mathrm{Fi}$, microwave oven, cordless phone, bluetooth, and ZigBee. Such problem has to be tackled and dissected when implementing the smart energy for home automation.

2) Interoperability: Some end devices in heterogeneous networks may not be uniquely assigned with global IP addresses. Hence, they will require middlewares or convergence sublayers implemented in the gateways or powerful edge routers to be able to communicate among each other. The converged M2M gateway is able to perform protocol translation for different communications technologies, e.g., PLC with IEEE 802.15.4 proposed in [111]. The powerful edge devices are designed to efficiently surmount the issues of resource allocation and management, scheduling, admission control, as well as mobility with handover. Examples can be found in WiFi \& WiMAX integration for telemedicine scenario introduced in [112] and IEEE 802.21 media independent handover (MIH) approach proposed in [113].

On the other hand, interoperability raises a dilemma about whether to assign a global IPv6 address to every end device or implement a convergence sublayer for the protocol/address conversion, e.g., the Network Address Translation (NAT) used in IPv4 networks. Employing either one of them would be based upon the market needs as well as the strategies and requirements of service providers and utilities. For the overall network performance of the smart grid, it requires further investigation to achieve high throughput and low latency.

3) IPv6 Integration: The Internet of things encompasses hundreds of millions IP-based and Internet-connected embedded devices (i.e., smart objects) and networks [114]. The so-called low-power-RF-plus-IPv6 6LoWPAN defined in RFC4919 [115] and RFC4944 [116] with the associated Working Group: routing over low-power and lossy networks (WG-ROLL) formed in 2008 by Internet Engineering Task Force (IETF) has gained much attention nowadays. The lowpower and low-data-rate network will play an important role for applications of wireless embedded technology in the 
TABLE II

POTENTIAL TECHNOLOGIES SUPPORTING THE SMART GRID COMMUNICATIONS.

Communications

\begin{tabular}{|c|c|c|c|c|}
\hline & & & & \\
\hline \multirow[t]{3}{*}{$\begin{array}{r}\text { Wireless } \\
\text { Technologies }\end{array}$} & $\begin{array}{l}\text { Cellular } \\
(\text { GPRS/3G/4G) and } \\
\text { LTE }\end{array}$ & $\begin{array}{l}\text { Voice-initiated; Remote monitoring } \\
\text { and control (e.g., SCADA) for } \\
\text { substations and distributed energy } \\
\text { sources; Simple text messaging } \\
\text { support }\end{array}$ & $\begin{array}{l}\text { Low implementation, opera- } \\
\text { tional, and maintenance costs } \\
\text { using existing network infra- } \\
\text { structures; Larger coverage; } \\
\text { Better roaming and mobility } \\
\text { support }\end{array}$ & $\begin{array}{l}\text { Need for towers/base stations; Un- } \\
\text { economical call establishment on } \\
\text { large scales; Unavailable coverage } \\
\text { for some remote sites; Security- } \\
\text { vulnerable }\end{array}$ \\
\hline & WiFi (IEEE 802.11) & $\begin{array}{l}\text { Data (and video)-initiated; Home } \\
\text { energy interface; Connection among } \\
\text { PCs, laptops, PDAs, and customer } \\
\text { electronics, as well as smart meter- } \\
\text { ing solutions }\end{array}$ & $\begin{array}{l}\text { Rapid installation; High flex- } \\
\text { ibility; Solutions for aggrega- } \\
\text { tion points in urban areas }\end{array}$ & $\begin{array}{l}\text { High interference-sensitivity; Small } \\
\text { coverage; Power-hungry; Uneconom- } \\
\text { ical on small scales; Security- } \\
\text { vulnerable }\end{array}$ \\
\hline & $\begin{array}{l}\text { WiMAX (IEEE } \\
802.16)\end{array}$ & $\begin{array}{l}\text { Last-mile wireless broadband } \\
\text { access alternative to Cable and } \\
\text { DSL; Smart metering network in } \\
\text { AMI }\end{array}$ & $\begin{array}{l}\text { Fast deployment when com- } \\
\text { pared to wired solutions; } \\
\text { Long-range; High speed for } \\
\text { real-time applications and fast } \\
\text { response }\end{array}$ & $\begin{array}{l}\text { Need for towers/base stations; Low } \\
\text { penetration while operating in very } \\
\text { high frequency bands; High power } \\
\text { consumption; Security-vulnerable }\end{array}$ \\
\hline \multirow[t]{2}{*}{$\begin{array}{r}\text { Wireline } \\
\text { Technologies }\end{array}$} & $\begin{array}{l}\text { SONET/SDH and } \\
\text { E/GPON }\end{array}$ & $\begin{array}{l}\text { Fiber optics-based; Broadband } \\
\text { solutions for core, metro, and access } \\
\text { networks }\end{array}$ & $\begin{array}{l}\text { High bandwidth and large } \\
\text { capacity support; Fast trans- } \\
\text { mission; Negligible interfe- } \\
\text { rence }\end{array}$ & $\begin{array}{l}\text { Slow deployment and high cost in- } \\
\text { stallation if no existing infrastructure } \\
\text { available especially in rural areas }\end{array}$ \\
\hline & $\begin{array}{l}\text { PLC }(\mathrm{NB} \text { and } \mathrm{BB}) \\
\text { and } \mathrm{BPL}\end{array}$ & $\begin{array}{l}\text { Power-initiated; Particular commu- } \\
\text { nication channels in MV and LV } \\
\text { fields; BPL broadband access alter- } \\
\text { native to Cable and DSL }\end{array}$ & $\begin{array}{l}\text { Complementing cable and } \\
\text { wireless solutions; Easy in- } \\
\text { stallation for indoors; Higher } \\
\text { flexibility and mobility for } \\
\text { end devices; Solutions for } \\
\text { rural areas }\end{array}$ & $\begin{array}{l}\text { Complex implementation for larger } \\
\text { buildings; Phase switch challenge } \\
\text { from indoor to outdoor and vice } \\
\text { versa; Signal attenuation and high } \\
\text { cost for repeaters deployment in } \\
\text { localized areas; High interference } \\
\text { over power lines }\end{array}$ \\
\hline \multirow[t]{2}{*}{$\begin{array}{r}\text { Network } \\
\text { Types }\end{array}$} & WMN & $\begin{array}{l}\text { Mesh network supported in com- } \\
\text { munities and neighborhoods; Super } \\
\text { mesh routers managing diverse } \\
\text { applications }\end{array}$ & $\begin{array}{l}\text { Easy and cost-effective instal- } \\
\text { lation; High reliability and } \\
\text { flexibility; Self-configuration } \\
\text { and healing }\end{array}$ & $\begin{array}{l}\text { High complexity in data manage- } \\
\text { ment; Low controllability in unli- } \\
\text { censed spectrums; Lack of standards; } \\
\text { Overheads }\end{array}$ \\
\hline & $\begin{array}{l}\text { WSN and WPAN } \\
\text { (IEEE 802.15.4) }\end{array}$ & $\begin{array}{l}\text { Small measures; Home, office, and } \\
\text { smart appliance (energy) automa- } \\
\text { tion; Sensing, monitoring, control in } \\
\text { fields of substations, industrial } \\
\text { facilities, and distributed generation }\end{array}$ & $\begin{array}{l}\text { Easy and rapid deployment; } \\
\text { Low cost; High portability; } \\
\text { Easy configuration }\end{array}$ & $\begin{array}{l}\text { Power and memory constrained; Low } \\
\text { data rate; Higher data loss; Very low } \\
\text { coverage }\end{array}$ \\
\hline Proprietary & Dedicated or Private & $\begin{array}{l}\text { Pre-assigned and possession of } \\
\text { mixed telecommunications technol- } \\
\text { ogies; Licensed spectrums }\end{array}$ & $\begin{array}{l}\text { Less security-vulnerable; No } \\
\text { sharing in bandwidth as well } \\
\text { as profits on capitals; Higher } \\
\text { independence }\end{array}$ & $\begin{array}{l}\text { Lower flexibility and manageability; } \\
\text { Very high installation cost }\end{array}$ \\
\hline
\end{tabular}

foreseeable future. Examples for such type of networks may include [114], [117]

- Network devices based on IEEE 802.15.4, e.g., ZigBee, ISA100.11a, IP500 Alliance;

- Networks using sub-GHz band radios (i.e., frequencies bellow $1 \mathrm{GHz}$ proposed in IEEE 802.15.4g and IEEE $802.11 \mathrm{ah}$ ) as communications channels for better RF penetration as well as less-severe interference;

- The PLC-based Watteco's Watt Pulse Communication (WPC) as well as HomePlug Green.

Figure 8 depicts an example of the communications layer of integrating IP in HAN via the 6LoWPAN mechanism.

The interaction of diverse technologies in physical (PHY) and medium access control (MAC) layers using IP for the protocols convergence on top of link layer will enhance the efficiency and reliability of data transport. In the network layer, Internet control message protocol (ICMP) is fundamentally available for network diagnosis and routing purposes. The routing scheme named routing protocol for low power lossy networks (RPL) is also supported for the IP mesh networking of 6LoWPAN defined by WG ROLL. End-to-end transport protocols such as user datagram protocol (UDP), transmission

\begin{tabular}{|c|c|}
\hline \multicolumn{2}{|c|}{$\begin{array}{c}\text { Application, Presentation, Session } \\
\text { Layers (applications) }\end{array}$} \\
\hline UDP, TCP, SCTP & $\begin{array}{c}\text { Transport } \\
\text { Layer }\end{array}$ \\
\hline $\begin{array}{c}\text { IPv6, 6LoWPAN, RPL } \\
\text { ICMP }\end{array}$ & $\begin{array}{c}\text { Network } \\
\text { Layer }\end{array}$ \\
\hline $\begin{array}{c}\text { 802.15.4, 802.11 } \\
\text { PLC, HomePlug }\end{array}$ & $\begin{array}{c}\text { Data Link } \\
\text { Layer }\end{array}$ \\
\hline $\begin{array}{c}\text { 802.15.4, 802.11 } \\
\text { PLC, HomePlug }\end{array}$ & $\begin{array}{c}\text { Physical } \\
\text { Layer }\end{array}$ \\
\hline \hline
\end{tabular}

Fig. 8. Various technologies in PHY/MAC layers utilizing IP for efficient data transport.

control protocol (TCP) [118], and stream control transmission protocol (SCTP) are optionally employed depending upon types of data and applications, as well as power and complexity constraints in the networks. 


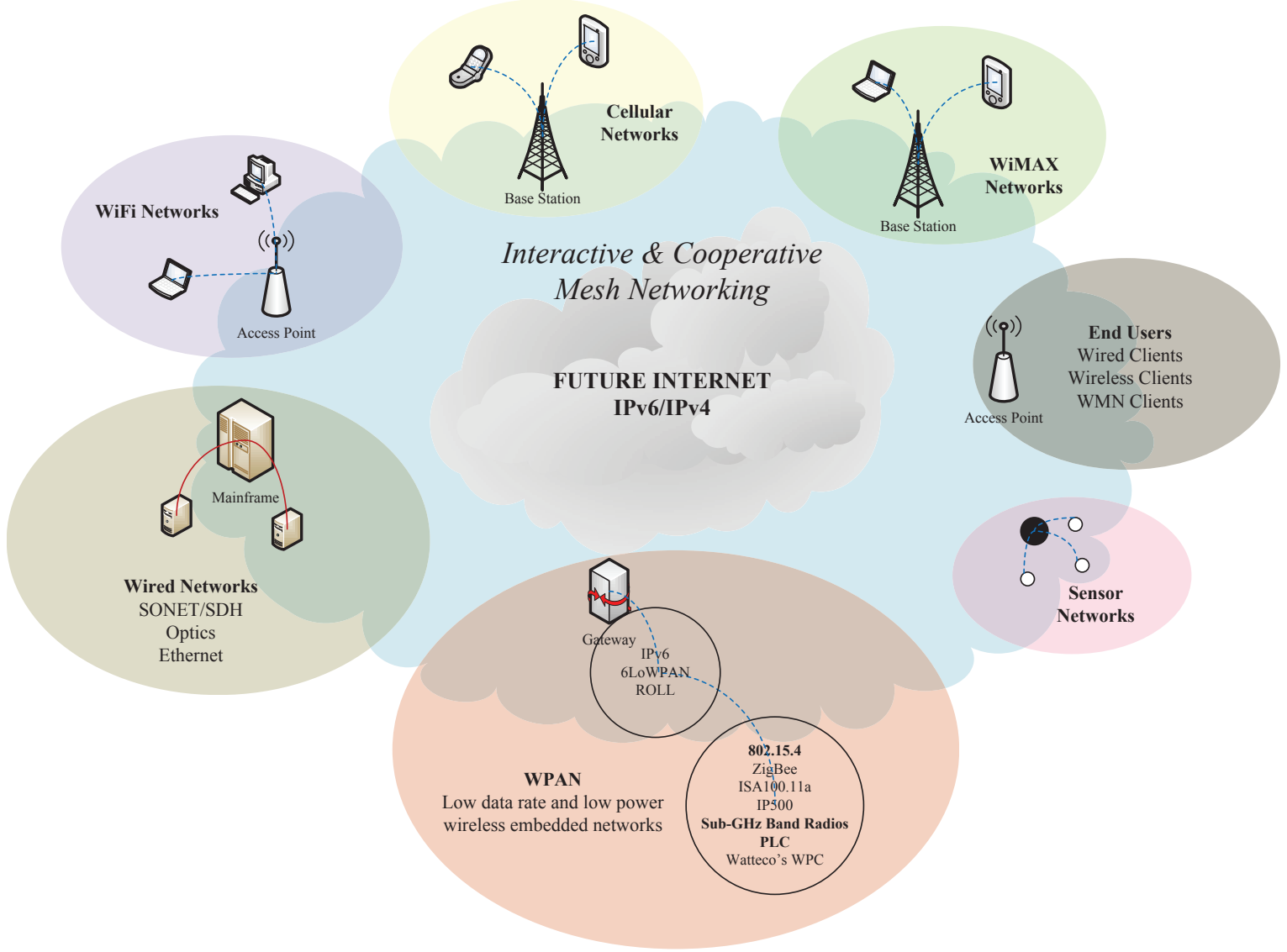

Fig. 9. The interactive wireless mesh networking infrastructure with the convergence of the LPLR wireless embedded networks via 6LoWPAN.

The main benefit of 6LoWPAN is the ease of connecting logical-addressed devices to the global IP networks without adding additional translating gateways or proxies. Nonetheless, this entails a list of challenges:

- Header compression and packets fragmentation due to the minimum frame length or maximum transmission unit (MTU) of 1280-Byte for IPv6;

- The consequent firewall and NAT issues;

- Vulnerability-prone security that is only based on advanced encryption standard (AES) in the MAC layer;

- Support for IPv4, i.e., IPv6-in-IPv4 tunneling and address translation;

- Neighbor discovery and routing requirements.

Readers are referred to [119] and [120] for further investigation.

IP has great potentials to coalesce a number of diverse communications protocols for the smart grid [19], [121]. The de facto standard has existed since 1980s. Although it is a publicly open communications protocol (which may seem to be vulnerable to attacks), it provides the most flexibility for its standard-upgrade as well as mature security/tools for securing and managing the data transport in both public and private networks. In fact, a number of financial networks in commerce and industries (e.g., banks, retail banking sections) today utilize IP as foundation to secure their networks. The solid examples can be tailored for the smart grid implementation. Cisco's smooth IP migration [121] uses IP to help different types of physical equipments to operate transparently from end
TABLE III

COMMUNICATIONS STANDARDS LIKELY TO BE DEPLOYED IN DIFFERENT COUNTRIES FOR HAN AND NAN/FAN OF THE SMART GRID

\begin{tabular}{|c|c|c|c|c|c|c|c|c|c|c|}
\hline \multicolumn{2}{|c|}{} & \multicolumn{3}{|c|}{$\mathbf{8 0 2 . 1 5 . 4}$} & \multicolumn{5}{c|}{ Sub-GHz } & \\
\hline \multirow{2}{*}{ HAN } & WiFi & ZigBee & $.4 \mathrm{~g}$ & HomePlug & PLC & ISM & Prop. & WiMAX & GPRS \\
\hline & EU & $\checkmark$ & $\checkmark$ & & $\checkmark$ & & $\checkmark$ & & & \\
\hline \multirow{3}{*}{ NAN } & & $\checkmark$ & & & $\checkmark$ & $\checkmark$ & & & \\
\hline & NA & & & $\checkmark$ & & & & $\checkmark$ & $\checkmark$ & \\
\hline & EU & & & & & $\checkmark$ & & $\checkmark$ & & $\checkmark$ \\
\hline & Asia & & & & & & & $\checkmark$ & & \\
\hline
\end{tabular}

to end over a variety of media without protocols conversion. The steps of IP convergence, from encapsulation, gateways, to native IP, were confidently presented in [121].

Table III shows exemplified territories in which various communications standards would likely be or have started to be implemented: North America will probably adopt Wi$\mathrm{Fi}$, ZigBee, HomePlug in HAN and WiMAX/802.15.4g in NAN. Meantime, parts of Europe favors PLC and GPRS in view of its legacy power infrastructure, whereas Australia uses WiMAX [122]. Noticeably, the types of material by which construction and buildings were built would affect the network performance especially in RF communications. Since buildings in Europe are mostly of stone or brick construction rather than wood frame, the RF technology such as IEEE 802.15.4 might not be a suitable solution for their HAN. Similarly, countries such as United States, Australia, and 


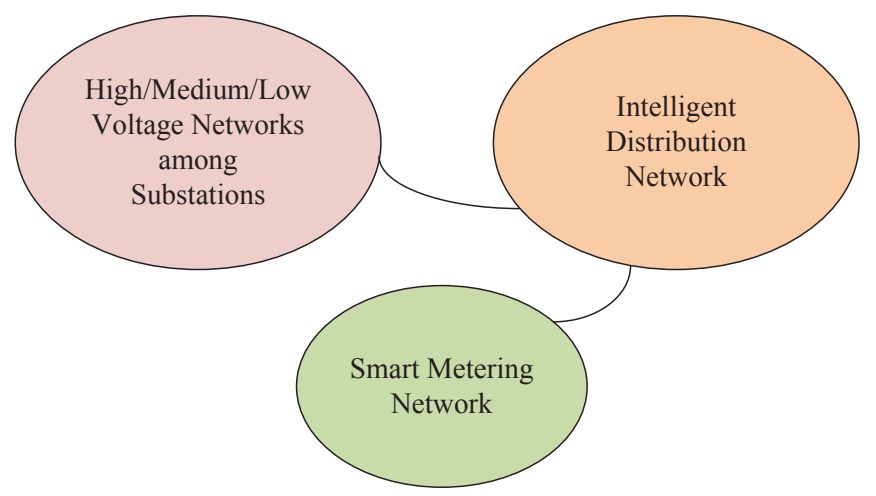

Fig. 10. Interconnecting communications networks in the Smart Grid system.

Russia have discontinued deploying PLC due to its prohibitive implementation cost [33].

The smart grid initiative is deemed to be the next driving force of interconnecting and tunneling communications protocols of heterogeneous networks over the common standard IP. It integrates both wireline and wireless technologies into one interactive end-to-end mesh network to support full coverage, flexibility, as well as mobility and roaming for the effective smart grid system.

As illustrated in Figure 9, the integrated system has prodigious potentials and is able to provide seamless and continuous services anytime and anywhere among different types of networks with various access technologies. The integration of WAN as well as NAN/FAN and HAN solutions not only will be vastly explored to support smart grid applications, security, and QoS, but also will bolster both wired and wireless broadband deliveries.

\section{Open Research Issues and Challenges}

The dramatic growth of network traffic and users has played up the importance of designing application-aware communications networks nowadays. A great challenge is to support a wide range of smart grid applications and requirements in two-way communications for the power grid system. Similar to various applications such as e-learning and advanced education, telemedicine, VoIP, and other business programs, massive sensors/actuators and IEDs and smart meters will soon generate an enormous amount of data for the entire smart grid operation. Figure 10 illustrates the three main networks with arising measuring devices added throughout the grid, typically for remote sensing for wide area monitoring and control in transmission networks, DMS/MDM/DSM in distribution networks, as well as power usage and saving via smart metering networks. The flood of raw data across heterogenous networking technologies requires comprehensive data management. Challenges include making good use of these data for expeditious subsequent actions, and capitalizing on these data to enhance smart grid capabilities.

The issue of proliferating various product manufacturers and power suppliers has brought standardization to the forefront of the smart grid implementation. Deploying standard communications technologies is preferable to proprietary solutions for utility providers [63]. Not only should compatibility and interoperability among communications technologies coexisting in the PHY/MAC layers be possibly made available, but also their data objects as well as information representation (e.g., DLMS or Device Language Message Specification [33], ZigBee, Wireless M-Bus) should be processed in the application layer on user-friendly interfaces. Both wireline and wireless technologies are complementary to the needs of the reliable smart grid communications network. For example, cooperation of HomePlug AV and ZigBee implemented in HAN as well as interconnection of WiMAX and LTE covering rural and urban areas communications in NAN/FAN will require extensive investigations.

While wireless communications will play the vital role in the smart grid system, management of radio spectrum needs to be reassessed and redesigned for its efficient use. Statistics have shown that radio spectrum/channel (one of the world's most valuable resources) is mostly underutilized [123]. Cognitive radio network (CRN) has been researched for years, though mostly focused on the PHY/MAC layers. The IEEE 802.22 wireless regional area network (WRAN) standard using white spaces in TV bands $(54-862 \mathrm{MHz})$ has been proposed for cognitive radio based operations. The active research area should be comprehensively explored in terms of how the unused spectrums can be dynamically detected from the primary user network (licensed) and allocated to the secondary user network (unlicensed) to support smart grid applications. Such mechanism involving intelligent spectrum sensing and scheduling has great potentials to mitigate the heavy medium contention and intra-system coexistence issues in the unlicensed ISM channels. It may also alleviate the burden of purchasing licensed spectra for utility providers who are concerned with the extra costs. In order to maximize the overall communications throughput and minimize its delay for efficient data transmission in the cognitive smart grid system, truly environmental-aware cross-layer interaction models from the physical to transport layer as well as application-aware communications protocols in wireless and hybrid wirelesswireline network architectures are needed. Meanwhile, green energy-efficient communications designs [124]-[126] should be well considered vertically across the OSI protocol layers, from the adaptive power control, flexible modulation and coding as well as network coding theory, innovative contention and hidden node collision avoidance (especially in IEEE802.15.4), acknowledgement schemes and error correction, holistic routing, to the sleep mode tactic under various smart grid application constrains and requirements.

Other challenges in enabling the power grid with telecommunications technologies also need attention, such as service coverage and bandwidth by $2 \mathrm{G} / 3 \mathrm{G}$ cellular networks, which were not fundamentally designed for communications of application awareness. A rise of the number of communicating devices will impact their network reliability and availability as well as channel assignment per cell. For the criticalinfrastructure smart grid system, channel availability under natural disaster or power outage scenarios must be guaranteed for emergencies. While the cellular and Wi-Fi networks provide best-effort services, the coming 4G LTE and advanced WiMAX should carefully address these issues in their middle 
development stage to support the grid communications.

Contrary to multimedia applications, smart grid applications communications are mostly (control) messages which are jitter-insensitive with small packet size. A new QoS-based communication protocol design for the smart grid should focus on types of data traffic, data prioritization, and application awareness. Furthermore, intelligent bandwidth scheduling and allocation for high resource utilization is required for different modes of data collection (fixed, event-driven, demand-driven) in AMI [33]. The transport protocol such as TCP should be redesigned to reduce latency and overhead in such small packet transmission. Meanwhile, UDP, SCTP, as well as other transport protocols can also be considered and further studied. From the security perspectives, lightweight encryption for smart grid data confidentiality and authentication for its integrity are desired. Notably, there has been a debate on whether or not the mesh network mechanism should be implemented among smart meters due to the consumers' privacy reason.

Finally, the data aggregation technique used in smart metering networks should be different from the ones proposed for sensors and actuators networks because each individual data information for energy usage needs to be preserved and collected. At the same time, the multiple-to-one traffic loads will require larger uplink bandwidths.

\section{CONCLUSION}

The idea of smart grid is referring to smart generation and consumption between energy suppliers and consumers. It aims to achieve reliability, efficiency, and optimization in operation and planning, demand response, as well as utilization of diverse resources. Such smart infrastructure and integration of advanced power production and delivery, metrology, communications technologies, and information, are vastly required to attain the ultimate target.

While enabling data information to help control energy usage throughout the system, uniting the smart grid agenda with a larger social vision of a better world is the key to winwin success eventually. Furthermore, educating people on use of technologies and on energy consumption and conservation is essential during the smart grid development stage.

The purpose of this article is to provide an elaborate tutorial on the smart grid blueprint for electric power system reform from both power and communications standpoints. It introduces what a smart grid is, why it is extremely desired, what can be done, and how it can be accomplished, explained in a coordinated contextual arrangement throughout the contents. A number of issues considered as triggers for enabling modernization in electricity economy and its infrastructure are addressed in Section I \& II. The capability and requirements that a smart grid should acquire are discussed predominantly in Section III. A variety of wireline and wireless technologies as well as types of networks for the smart grid communications infrastructure and its networking, followed by a discussion of foreseeable challenges, are conferred in Section IV.

A grand overarching strategy to construct the smart grid will fundamentally depend upon

- Drawn-up interoperable standards in both power and communications aspects;
- Advancement of electric power technologies and assets;

- Adequacy of communications technologies adoption, especially for distribution automation and management, as well as smart metering and home energy management systems;

- Effective and secure communications;

- Development and integration of RES and DG;

- Intelligence of practical and ancillary data and control management.

Conclusively, the modernized grid will continue to energize a tremendous amount of interest and creativity in different fields. It will further create innovative research on designing and building a robust, consistent, safe and secure community of the smart grid in the upcoming years.

\section{REFERENCES}

[1] L. Tsoukalas and R. Gao, "From Smart Grids to an energy Internet: Assumptions, architectures and requirements," in Proc. 3rd IEEE International Conference on Electric Utility Deregulation and Restructuring and Power Technologies (DRPT), Nanjing, China, 6-9 Apr. 2008, pp. 94-98.

[2] Smart Grid News.com. [Online]. Available: http://www. smartgridnews.com/

[3] (2006) GridWorks: Overview of the electric grid. U.S. Department of Energy (DOE) - Office of Electricity Delivery \& Energy Reliability. [Online]. Available: http://sites.energetics.com/gridworks/grid.html

[4] S. Spoonamore and R. L. Krutz. (2010, Jan.) Smart Grid and cyber challenges. [Online]. Available: http://www.whitehouse.gov/ files/documents/cyber/Spoonamore-Krutz $\% 20-\% 20$ Smart\%20Grid\%20CyberSecurity\%20Risks\%20and\%20 Concerns.pdf

[5] T. L. Friedman, Hot, Flat, and Crowded: Why We Need a Green Revolution and How It Can Renew America. New York: Farrar, Straus and Giroux, 2008.

[6] S. Collier, "Ten steps to a smarter grid," IEEE Ind. Appl. Mag., vol. 16, no. 2, pp. 62-68, Mar.-Apr. 2010

[7] L. Freris and D. Infield, Renewable Energy In Power Systems. UK: Wiley, 2008.

[8] J. See, W. Carr, and S. Collier, "Real time distribution analysis for electric utilities," in Proc. IEEE Rural Electric Power Conference, North Charleston, SC, 27-29 Apr. 2008, pp. B5-B5-8.

[9] U.S. Energy Information Administration (EIA) - Independent Statistics and Analysis. [Online]. Available: http://www.eia.doe.gov/

[10] "The Smart Grid: An introduction," Book publication, U.S. Department of Energy (DOE), 2008. [Online]. Available: http://www.oe.energy.gov/DocumentsandMedia/DOE_SG_Book_Single_Pages(1).pdf.

[11] R. DeBlasio and C. Tom, "Standards for the Smart Grid," in Proc. IEEE Energy 2030 Conference (ENERGY), Abu Dhabi, 4-5 Nov. 2008, pp. $1-7$.

[12] IEEE smart grid standards. IEEE Smart Grid. [Online]. Available: http://smartgrid.ieee.org/standards

[13] E. W. Gunther. (2009, 21-24 Sep.) Standards: Help or hindrance in Smart Grid deployments. EnerNex. GridWeek Conference in Washington, DC. [Online]. Available: http://www.pointview.com/data/2009/09/31/pdf/Erich-Gunther4476.pdf

[14] C. Strauss, Practical Electrical Network Automation and Communication Systems. Oxford: Newnes, 2003.

[15] K. Narendra and T. Weekes, "Phasor measurement unit (PMU) communication experience in a utility environment," Easun Reyrolle Ltd (ERL) Phase Power Technologies Ltd., CIGRE Conference on Power System, Tech. Rep., 19-21 Oct. 2008. [Online]. Available: http://www.erlphase.com/downloads/papers/08_CIGRE_PMU_Communication_Experience.pdf

[16] T. F. Garrity, "Getting smart," IEEE Power Energy Mag., vol. 6, no. 2, pp. 38-45, Mar.-Apr. 2008.

[17] R. James, "The power to reduce $\mathrm{CO}_{2}$ emissions: The full portfolio," Electric Power Research Institute (EPRI), Tech. Rep. 1020389, 26 Oct. 2009. [Online]. Available: http://my.epri.com/portal/server.pt?Abstract_id=000000000001020389 
[18] J. Polonetsky and C. Wolf. (2009, 3 Nov.) How privacy (or lack of it) could sabotage the grid. Smart Grid News.com. [Online]. Available: http://www.smartgridnews.com/artman/publish/Technologies_Metering News/

[19] C. D. Marson. (2009, 29 Oct.) Q\&A: Why IP is the right choice for Smart Grid. Network World. [Online]. Available: http://www.networkworld.com/news/2009/102909-smartgrid-ipv6- qa.html

[20] D. G. Hart, "Using AMI to realize the Smart Grid," in Proc. IEEE Power and Energy Society General Meeting - Conversion and Delivery of Electrical Energy in the 21st Century, Pittsburgh, PA, 20-24 July 2008, pp. 1-2.

[21] "Study of security attributes of Smart Grid systems: Current cyber security issues," Idaho National Laboratory (INL), National SCADA Test Bed (NSTB) Rep. INL/EXT-09-15500, Apr. 2009. [Online]. Available: http://www.inl.gov/scada/publications/d/securing_the_smart_grid current_issues.pdf

[22] "Enabling tomorrow's electricity system: Report of the ontario Smart Grid forum," Independent Electricity System Operator (IESO), Forum Rep., Feb. 2009. [Online]. Available: http://www.ieso.ca/imoweb/pubs/smart_grid/Smart_Grid_ForumReport .pdf

[23] A. Harris, "Smart Grid thinking - [power super grid]," IET/IEEE Eng. Technol., vol. 4, no. 9, pp. 46-49, 23 May-5 June 2009.

[24] C. Lima. (2010) Smart Grid communications: Enabling a smarter grid. IEEE SCV ComSoc Monthly Meeting. [Online]. Available: http://ewh.ieee.org/r6/scv/comsoc/Workshop_092510_EnablingSmarter Grid.pdf

[25] J. Belagur and R. Schmidt, "IP communication for substation automation, distribution automation, and other utility applications - a business case," in Proc. IEEE/PES Transmission and Distribution Conference and Exposition: $T \&$ D , Bogota, Colombia, 21-24 Apr. 2008, pp. 1-9.

[26] M. Vadiati, M. Ghorbani, A. Ebrahimi, and M. Arshia, "Future trends of substation automation system by applying IEC 61850," in Proc. 43rd IEEE International Universities Power Engineering Conference (UPEC), Padova, Italy, 1-4 Sept. 2008, pp. 1-4.

[27] C. LaPlace and R. W. Uluski. (2009, 30 Jan.) Realizing the Smart Grid of the future through AMI technology. Energyaxis powered by Elster. [Online]. Available: http://elsterenergyaxis.com/pdf/RealizingSmartGrid.pdf

[28] J. Fan and S. Borlase, "The evolution of distribution," IEEE Power Energy Mag., vol. 7, no. 2, pp. 63-68, Mar-Apr. 2009.

[29] L. Cao, J. Tian, and Y. Liu, "Remote wireless automatic meter reading system based on wireless mesh networks and embedded technology," in Proc. 5th IEEE International Symposium on Embedded Computing (SEC), Beijing, China, 6-9 Oct. 2008, pp. 192-197.

[30] B. Sivaneasan, P. So, and E. Gunawan, "Modeling and performance analysis of automatic meter reading systems using power line communications," in Proc. 11th IEEE Singapore International Conference on Communication Systems (ICCS), Guangzhou, China, 19-21 Nov. 2008, pp. $1446-1450$.

[31] E. Valigi and E. di Marino, "Networks optimization with advanced meter infrastructure and smart meters," in Proc. 20th IEEE International Conference and Exhibition on Electricity Distribution, Prague, Czech Republic, 8-11 June 2009, pp. 1-4.

[32] H. Y. Tung, K. F. Tsang, and K. L. Lam, "ZigBee sensor network for advanced metering infrastructure," in Proc. IEEE Digest of Technical Papers International Conference on Consumer Electronics (ICCE), Las Vegas, NV, 9-13 Jan. 2010, pp. 95-96.

[33] T. Khalifa, K. Naik, and A. Nayak, "A survey of communication protocols for automatic meter reading applications," IEEE Commun. Surveys Tutorials, vol. 13, no. 2, pp. 168-182, 2nd qtr. 2011.

[34] J. Jones, "Experience of great river energy with meter data management," in Proc. IEEE/PES Power Systems Conference and Exposition (PSCE), Seattle, WA, 15-18 Mar. 2009, pp. 1-1.

[35] A. Vos, "Demand response management systems: The next wave of Smart Grid software," in Electric Energy $T \& D$ Magazine, EONLINE, M. A. Marullo, Ed. Steven Desrochers, Sep.-Oct. 2009, pp. 35-37. [Online]. Available: http://www.electricenergyonline.com/?page=magazine $\& I D=59$

[36] Z. Jiang, F. Li, W. Qiao, H. Sun, H. Wan, J. Wang, Y. Xia, Z. Xu, and P. Zhang, "A vision of smart transmission grids," in Proc. IEEE Power \& Energy Society General Meeting (PES), Calgary, Alberta, Canada, 26-30 July 2009, pp. 1-10.

[37] G. Benysek, Improvement in the Quality of Delivery of Electrical Energy using Power Electronics Systems. London, UK: Springer, 2007.
[38] R. Strzelecki and G. Benysek, Power Electronics in Smart Electrical Energy Networks. London, UK: Springer, 2008.

[39] S. Christensen and B. Bujnowski, "Smart Grid strategies what they mean for the workforce," 10 Mar. 2010, webinar organized by Smart Grid News.com. [Online]. Available: http://www. smartgridnews.com/webinars/2010_03_10/2010_03_10_PPTX _SGN \%20_AccentureSlides_Final.ppt

[40] U.S. Environmental Protection Agency (EPA). [Online]. Available: http://www.epa.gov/

[41] (2010, Mar.) World wind energy report 2009. World Wind Energy Association (WWEA). [Online]. Available: http://www.wwindea.org/home/images/stories/worldwindenergyreport 2009_s.pdf

[42] (2010, 17 Mar.) Solarbuzz reports world solar photovoltaic market grew to 6.43 gigawatt in 2009. Solarbuzz. [Online]. Available: http://www.evwind.es/noticias.php?id_not=4692

[43] N. Lu, T. Taylor, W. Jiang, C. Jin, J. Correia, L. Leung, and P. C. Wong, "Climate change impacts on residential and commercial loads in the western u.s. grid," IEEE Trans. Power Syst., vol. 25, no. 1, pp. 480-488, Feb. 2010.

[44] L. Beard, J. Cardell, I. Dobson, F. Galvan, D. Hawkins, W. Jewell, M. Kezunovic, T. Overbye, P. Sen, and D. Tylavsky, "Key technical challenges for the electric power industry and climate change," IEEE Trans. Energy Convers., vol. 25, no. 2, pp. 465-473, June 2010.

[45] H. Khurana, M. Hadley, N. Lu, and D. Frincke, "Smart-grid security issues," IEEE Security Privacy, vol. 8, no. 1, pp. 81-85, Jan.-Feb. 2010.

[46] F. Cleveland, "Cyber security issues for advanced metering infrastructure (AMI)," in Proc. IEEE Power and Energy Society General Meeting - Conversion and Delivery of Electrical Energy in the 21st Century, Pittsburgh, PA, 20-24 July 2008, pp. 1-5.

[47] T. G. Lewis, Critical Infrastructure Protection in Homeland Security: Defending a Networked Nation. Hoboken, NJ: Wiley, 2006.

[48] J. R. Bryan. (2009, 27 May) Utility's perspective of Smart Grid. Xcel Energy. [Online]. Available: http://www. johnrbryan. com/John_R_Bryan/Smart_Grids/Entries/2009/5/25_Smart_Grid_-_Utilitys_Perspective_Presentation_at_SK_Groups_Green_Days_files/ Smart\%20Grid\%20-\%20Micro\%20vs\%20Macro.pdf

[49] "National action plan on demand response," The Federal Energy Regulatory Commission (FERC), Draft Rep. for Comment AD09-10, 11 Mar. 2010. [Online]. Available: http://www.ferc.gov/legal/staffreports/03-12-10-demand-response.pdf

[50] IntelliGrid publications. Electric Power Research Institute (EPRI). [Online]. Available: http://intelligrid.epri.com/publications.html

[51] (2009, 19 Nov.) Handbook for assessing Smart Grid projects. GridWise Alliance. [Online]. Available: http://www.gridwise.org/documents/MetricsReportFinalEditionAll comments 11.30.09vFINALCOPYVERSION.pdf

[52] B. Blessing, "Smart Grid networking," White paper, Burns \& McDonnell, 16 Mar. 2010. [Online]. Available: http://www. burnsmcd.com/portal/page/portal/Internet/Content_Admin/Publi cations\%20Repository/Others\%20Link\%20Repository/WhitePaperSmart-Grid-Networking-March-2010-Blessing.pdf

[53] Y. Serizawa, E. Ohba, and M. Kurono, "Present and future ICT infrastructures for a smarter grid in Japan," in Proc. IEEE Innovative Smart Grid Technologies (ISGT), Gaithersburg, MD, 19-21 Jan. 2010, pp. 1-5.

[54] S.-Y. Son and B.-J. Chung, "A Korean Smart Grid architecture design for a field test based on power IT," in Proc. IEEE Transmission Distribution Conference Exposition: Asia and Pacific, Seoul, Korea, 26-30 Oct. 2009, pp. 1-4.

[55] J. Lu, D. Xie, and Q. Ai, "Research on Smart Grid in China," in Proc. IEEE Transmission Distribution Conference Exposition: Asia and Pacific, Seoul, Korea, 26-30 Oct. 2009, pp. 1-4.

[56] Q. Liu, B. Zhao, Y. Wang, and J. Hu, "Experience of AMR systems based on BPL in China," in Proc. IEEE International Symposium on Power Line Communications and Its Applications (ISPLC), Dresden, Germany, 29 Mar.-1 Apr. 2009, pp. 280-284.

[57] F. Lobo, A. Lopez, A. Cabello, D. Mora, R. Mora, F. Carmona, J. Moreno, D. Roman, A. Sendin, and I. Berganza, "How to design a communication network over distribution networks," in Proc. 20th IEEE International Conference and Exhibition on Electricity Distribution, Prague, Czech Republic, 8-11 June 2009, pp. 1-4.

[58] E. Peeters, R. Belhomme, C. Batlle, F. Bouffard, S. Karkkainen, D. Six, and M. Hommelberg, "ADDRESS: Scenarios and architecture for active demand development in the Smart Grids of the future," in Proc. 20th IEEE International Conference and Exhibition on Electricity Distribution, Prague, Czech Republic, 8-11 June 2009, pp. 1-4. 
[59] (2009, 25 Sept.) The InovGrid Project: Distribution network evolution as a decisive answer to the new challenges in the electrical sector. European Commission Research. [Online]. Available: http://ec.europa.eu/research/conferences/2009/smart_networks/pdf/messias.pdf

[60] (2010, 10 Feb.) The 2010 North American utility Smart Grid deployment survey. GreenTechMedia Research. [Online]. Available: http://www.gtmresearch.com/report/the-2010-northamerican-utility- smart-grid-deployment-survey

[61] H. Khan, Z. Xu, H. Iu, and V. Sreeram, "Review of technologies and implementation strategies in the area of Smart Grid," in Proc. 19th Australasian Universities Power Engineering Conference (AUPEC), Adelaide, Australia, 27-30 Sept. 2009, pp. 1-6.

[62] J. G. Cupp and M. E. Beehler, "Implementing Smart Grid communications: Managing mountains of data opens up new challenges for electric utilities," Burns \& McDonnell TechBriefs, no. 4, pp. 5-8, 2008. [Online]. Available: http://www.burnsmcd.com/portal/page/portal/Internet/Content_Admin/ Compl ete\%20Issues\%20Link\%20Repository/TB2008v4.pdf

[63] A. Clark and C. J. Pavlovski, "Wireless networks for the smart energy grid: Application aware networks," in Proc. International MultiConference of Engineers and Computer Scientists (IMECS), 17-19 Mar. 2010, pp. 1243-1248.

[64] M. Blatt. (2010, 1 Aug.) Next-Generation utility telecommunication solutions for the Smart Grid. Electric Light\&Power. [Online]. Available: http://www.elp.com/index/display/articledisplay/367600/articles/utility-products/volume-6/issue8/features/feature- story/next-generation-utility-telecommunicationsolutions-for-the-smart-grid.html

[65] J. Zhang and N. Ansari, "Scheduling hybrid WDM/TDM passive optical networks with nonzero laser tuning time," IEEE/ACM Transactions on Networking, to appear.

[66] J. Zhang, N. Ansari, Y. Luo, F. Effenberger, and F. Ye, "Nextgeneration PONs: A performance investigation of candidate architectures for next-generation access stage 1," IEEE Communications Magazine, vol. 47, no. 8, pp. 49-57, Aug. 2009.

[67] G. Bumiller, L. Lampe, and H. Hrasnica, "Power line communication networks for large-scale control and automation systems," IEEE Commun. Mag., vol. 48, no. 4, pp. 106-113, Apr. 2010.

[68] M. Yousuf and M. El-Shafei, "Power line communications: An overview - Part I," in Proc. 4th IEEE International Conference on Innovations in Information Technology (IIT), Dubai, United Arab Emirates, 18-20 Nov. 2007, pp. 218-222.

[69] A. Sarafi, G. Tsiropoulos, and P. Cottis, "Hybrid wireless-broadband over power lines: A promising broadband solution in rural areas," IEEE Commun. Mag., vol. 47, no. 11, pp. 140-147, Nov. 2009.

[70] S. Bannister and P. Beckett, "Enhancing powerline communications in the "Smart Grid" using OFDMA," in Proc. 19th Australasian Universities Power Engineering Conference (AUPEC), Adelaide, Australia, 27-30 Sept. 2009, pp. 1-5.

[71] M. Yousuf, S. Rizvi, and M. El-Shafei, "Power line communications: An overview - Part II," in Proc. 3rd IEEE International Conference on Information and Communication Technologies: From Theory to Applications (ICTTA), Damascus, Syria, 7-11 Apr. 2008, pp. 1-6.

[72] G. Held, Understanding Broadband Over Power Line. Boca Raton, FL: AUERBACH, 2006.

[73] R. Heickero, S. Jelvin, and B. Josefsson, "Ericsson seamless network," Ericsson Review, no. 2, pp. 76-83, 2002. [Online]. Available: http://www1.ericsson.com/ericsson/corpinfo/publications/review/2002_ 02/files/2002024.pdf

[74] Wireless LAN Medium Access Control (MAC) and Physical Layer (PHY) Specifications, IEEE Std. 802.11 ${ }^{\mathrm{TM}}, 2007$.

[75] (2009, Sept.) Wi-Fi CERTIFIED ${ }^{\mathrm{TM}} \mathrm{n}$ : Longer-range, faster-throughput, multimedia-grade Wi-Fi networks. The Wi-Fi Alliance. Amendment to IEEE Std 802.11 ${ }^{\mathrm{TM}}-2007$. [Online]. Available: http://www.wifi.org/register.php?file=wp_Wi-Fi_CERTIFIED_n_In- dustry.pdf

[76] C. Behroozi. (2008, 15 Dec.) Wireless mesh networks and the smart grid. Google TechTalks. [Online]. Available: http://www.youtube.com/watch?v=09dhjDcaT7g

[77] J. Yick, B. Mukherjee, and D. Ghosal, "Wireless sensor network survey," Computer Networks, vol. 52, no. 12, pp. 2292-2330, 2008.

[78] K. Fowler, "The future of sensors and sensor networks survey results projecting the next 5 years," in Proc. IEEE Sensors Applications Symposium (SAS), New Orleans, LA, 17-19 Feb. 2009, pp. 1-6.

[79] B. Sundararaman, U. Buy, and A. D. Kshemkalyani, "Clock synchronization for wireless sensor networks: a survey," Ad Hoc Networks, vol. 3 , no. 3 , pp. 281-323, 2005.
[80] G. Anastasi, M. Conti, M. D. Francesco, and A. Passarella, "Energy conservation in wireless sensor networks: A survey," Ad Hoc Networks, vol. 7, no. 3, pp. 537-568, 2009.

[81] I. Demirkol, C. Ersoy, and F. Alagoz, "MAC protocols for wireless sensor networks: a survey," IEEE Commun. Mag., vol. 44, no. 4, pp. 115-121, Apr. 2006.

[82] M. Ali, A. Bohm, and M. Jonsson, "Wireless sensor networks for surveillance applications - a comparative survey of MAC protocols," in Proc. 4th IEEE International Conference on Wireless and Mobile Communications (ICWMC), Athens, Greece, 27 Jul.-1 Aug. 2008, pp. 399-403.

[83] H. Luo, Y. Liu, and S. Das, "Routing correlated data in wireless sensor networks: A survey," IEEE Network, vol. 21, no. 6, pp. 40-47, Nov.Dec. 2007.

[84] H. Nakayama, N. Ansari, A. Jamalipour, and N. Kato, "Fault-resilient sensing in wireless sensor networks," Computer Communications, vol. 30, no. 11-12, pp. 2375-2384, September 2007, Special Issue on Security on Wireless Ad Hoc and Sensor Networks.

[85] E. Fasolo, M. Rossi, J. Widmer, and M. Zorzi, "In-network aggregation techniques for wireless sensor networks: a survey," IEEE Wireless Commun., vol. 14, no. 2, pp. 70-87, Apr. 2007.

[86] Z. Wang, L. Liu, M. Zhou, and N. Ansari, "A position-based clustering technique for ad hoc intervehicle communication," IEEE Trans. Syst., Man, and Cybern. C, Appl. Rev., vol. 38, no. 2, pp. 201-208, Mar. 2008.

[87] C. Zhang, E. Hou, and N. Ansari, "Node clustering in wireless sensor networks," in Wireless Sensor Networks: A Networking Perspective, J. Zheng and A. Jamalipour, Eds. Hoboken, NJ: Wiley/IEEE Press, Sept. 2009, ch. 6, pp. 173-214.

[88] A. Ghosh and S. K. Das, "Coverage and connectivity issues in wireless sensor networks: A survey," Pervasive and Mobile Computing, vol. 4, no. 3, pp. 303-334, 2008.

[89] K. Miyao, H. Nakayama, N. Ansari, and N. Kato, "LTRT: An efficient and reliable topology control algorithm for ad-hoc networks," IEEE Trans. Wireless Commun., vol. 8, no. 12, pp. 6050-6058, Dec. 2009.

[90] S. Misra, M. Reisslein, and G. Xue, "A survey of multimedia streaming in wireless sensor networks," IEEE Commun. Surveys Tutorials, vol. 10 , no. 4, pp. 18-39, 4th qtr. 2008.

[91] M. Kobayashi, H. Nakayama, N. Ansari, and N. Kato, "Reliable application layer multicast over combined wired and wireless networks," IEEE Trans. Multimedia, vol. 11, no. 8, pp. 1466-1477, Dec. 2009.

[92] D. Gozupek, S. Papavassiliou, and N. Ansari, "Enhancing quality of service provisioning in wireless ad hoc networks using service vector paradigm: Research articles," Journal of Wireless Communications and Mobile Computing, vol. 6, no. 7, pp. 1003-1015, 2006, Special Issue on Wireless Ad hoc Networks: Technologies and Challenges.

[93] N. Li, N. Zhang, S. K. Das, and B. Thuraisingham, "Privacy preservation in wireless sensor networks: A state-of-the-art survey," Ad Hoc Networks, vol. 7, no. 8, pp. 1501-1514, 2009, Privacy and Security in Wireless Sensor and Ad Hoc Networks.

[94] X. Chen, K. Makki, K. Yen, and N. Pissinou, "Sensor network security: a survey," IEEE Commun. Surveys Tutorials, vol. 11, no. 2, pp. 52-73, 2nd qtr. 2009.

[95] P. Sakarindr and N. Ansari, "Security services in group communications over wireless infrastructure, mobile ad hoc, and wireless sensor networks," IEEE Wireless Commun., vol. 14, no. 5, pp. 8-20, Oct. 2007.

[96] P. Baronti, P. Pillai, V. W. Chook, S. Chessa, A. Gotta, and Y. F. Hu, "Wireless sensor networks: A survey on the state of the art and the 802.15.4 and ZigBee standards," Computer Communications, vol. 30, no. 7, pp. 1655-1695, 2007, wired/Wireless Internet Communications.

[97] J. Song, S. Han, A. Mok, D. Chen, M. Lucas, and M. Nixon, "WirelessHART: Applying wireless technology in real-time industrial process control," in Proc. IEEE Real-Time and Embedded Technology and Applications Symposium (RTAS), St. Louis, MO, 22-24 Apr. 2008, pp. 377-386.

[98] T. Lennvall, S. Svensson, and F. Hekland, "A comparison of WirelessHART and ZigBee for industrial applications," in Proc. IEEE International Workshop on Factory Communication Systems (WFCS), Dresden, Germany, 20-23 May 2008, pp. 85-88.

[99] K. Al Agha, M.-H. Bertin, T. Dang, A. Guitton, P. Minet, T. Val, and J.-B. Viollet, "Which wireless technology for industrial wireless sensor networks? the development of OCARI technology," IEEE Trans. Ind. Electron., vol. 56, no. 10, pp. 4266-4278, Oct. 2009.

[100] V. Gungor and G. Hancke, "Industrial wireless sensor networks: Challenges, design principles, and technical approaches," IEEE Trans. Ind. Electron., vol. 56, no. 10, pp. 4258-4265, Oct. 2009. 
[101] "Making the grid smarter," White paper, ECHELON, 2009. [Online]. Available: http://www.echelon.com/ solutions/smartgrid/documents/makingthegrid smarter.pdf

[102] "FireWire reference tutorial," White paper, 1394 Trade Association, 22 Jan. 2010. [Online]. Available: http://www. 1394ta.org/press/WhitePapers/Firewire\%20Reference\%20 Tutorial.pdf

[103] K. Gill, S.-H. Yang, F. Yao, and X. Lu, "A ZigBee-based home automation system," IEEE Trans. Consum. Electron., vol. 55, no. 2, pp. 422-430, May 2009.

[104] H. Park, M. Choi, E.-H. Paik, and N. Kim, "Interoperability model for devices over heterogeneous home networks," IEEE Trans. Consum. Electron., vol. 55, no. 3, pp. 1185-1191, Aug. 2009.

[105] C.-Y. Chen, Y.-P. Tsoul, S.-C. Liao, and C.-T. Lin, "Implementing the design of smart home and achieving energy conservation," in Proc. 7th IEEE International Conference on Industrial Informatics (INDIN), Cardiff, UK, 24-26 June 2009, pp. 273-276.

[106] J. Cheng and T. Kunz, "A survey on smart home networking," Carleton University, Systems and Computer Engineering, Tech. Rep. SCE-09-10, Sept. 2009. [Online]. Available: http://kunzpc.sce.carleton.ca/Thesis/SmartHomeNetworking.pdf

[107] IEEE 802.15: Wireless personal area networks (PANs). IEEE Standards Association. [Online]. Available: http://standards.ieee.org/getieee802/802.15.html

[108] K. Shuaib, M. Alnuaimi, M. Boulmalf, I. Jawhar, F. Sallabi, and A. Lakas, "Performance evaluation of IEEE 802.15.4: Experimental and simulation results," Academy Publisher Journal of Communications, vol. 2, no. 4, 2007.

[109] W. Yuan, X. Wang, and J.-P. Linnartz, "A coexistence model of IEEE 802.15.4 and IEEE 802.11b/g," in Proc. 14th IEEE Symposium on Communications and Vehicular Technology in the Benelux, Delft, Netherlands, 15 Nov. 2007, pp. 1-5.

[110] S. Pollin, I. Tan, B. Hodge, C. Chun, and A. Bahai, "Harmful coexistence between 802.15.4 and 802.11: A measurement-based study," in Proc. 3rd IEEE International Conference on Cognitive Radio Oriented Wireless Networks and Communications (CrownCom), Singapore, 1517 May 2008, pp. 1-6.

[111] J. Y. Ha, J. Jeon, K. Lee, J. Heo, N. Kim, S. M. Kim, W. H. Kwon, and B. J. Jung, "Design and implementation of convergence sub-layer for a heterogeneous home network," in Proc. IEEE International Symposium on Power Line Communications and Its Applications (ISPLC), Pisa, Italy, 26-28 Mar. 2007, pp. 252-256.

[112] Y. Zhang, N. Ansari, and H. Tsunoda, "Wireless telemedicine services over integrated IEEE 802.11/WLAN and IEEE 802.16/WiMAX networks," IEEE Wireless Commun., vol. 17, no. 1, pp. 30-36, Feb. 2010.

[113] O. Fratu, E. Popovici, S. Halunga, and E. Stanescu, "Consideration on interoperability of different wireless access networks using the IEEE 802.21 approach," in Proc. 9th IEEE International Conference on Telecommunication in Modern Satellite, Cable, and Broadcasting Services (TELSIKS), Nis, Serbia, 7-9 Oct. 2009, pp. 107-116.

[114] Z. Shelby and C. Bormann, 6LoWPAN: The Wireless Embedded Internet. Torquay, UK: Wiley, John \& Sons, 2009.

[115] N. Kushalnagar, G. Montenegro, and C. P. Schumacher, "IPv6 over low-power wireless personal area networks (6LoWPANs): Overview, assumptions, problem statement, and goals," Request for Comments (RFC): 4919, Aug. 2007. [Online]. Available: http://tools.ietf.org/html/rfc4919

[116] G. Montenegro, N. Kushalnagar, J. W. Hui, and D. E. Culler, "Transmission of IPv6 packets over IEEE 802.15.4 networks," Request for Comments (RFC): 4944, Sept. 2007. [Online]. Available: http://tools.ietf.org/html//rfc4944

[117] "Watteco's WPC: smart, safe, reliable and low power automation for home," White paper, Watteco, Dec. 2008. [Online]. Available: http://www.watteco.com/

[118] Y. Tian, K. Xu, and N. Ansari, "TCP in wireless environments: problems and solutions," IEEE Communications Magazine, vol. 43, no. 3, pp. S27-S32, Mar. 2005.

[119] A. Hasbollah, S. Ariffin, and M. Hamini, "Performance analysis for 6LoWPAN IEEE 802.15.4 with IPv6 network," in Proc. IEEE Region 10 Conference (TENCON), Singapore, 23-26 Nov. 2009, pp. 1-5.

[120] F. Lo Piccolo, D. Battaglino, L. Bracciale, M. Di Filippo, A. Bragagnini, M. Turolla, and N. Blefari Melazzi, "Towards fully IP-enabled IEEE 802.15.4 LR-WPANs," in Proc. 6th Annual IEEE Communications Society Conference on Sensor, Mesh and Ad Hoc Communications and Networks Workshops (SECON), Rome, Italy, 22-26 June 2009, pp. $1-3$.

[121] "Why IP is the right foundation for the Smart Grid," White paper, Cisco Systems, Jan. 2010. [Online]. Available: http://www.cisco.com/web/strategy/docs/energy/c11-581079_wp.pdf

[122] D. Imam and M. Dow. (2010) Communications flexibility is the key to an interoperable "Smart" Grid. TechBites Inc. [Online]. Available: http://www.techbites.com/

[123] B. Wang and K. Liu, "Advances in cognitive radio networks: A survey," IEEE J. Sel. Topics Signal Process., vol. 5, no. 1, pp. 5-23, Feb. 2011.

[124] T. Han and N. Ansari, "Energy efficient wireless multicasting," IEEE Communications Letters, vol. 15, no. 6, pp. 620-622, June 2011.

[125] J. Zhang and N. Ansari, "Toward energy-efficient 1G-EPON and 10G-EPON with sleep-aware MAC control and scheduling," IEEE Communications Magazine, vol. 49, no. 2, pp. s33-s38, Feb. 2011.

[126] N. Wang and N. Ansari, "Downloader-initiated random linear network coding for peer-to-peer file sharing," IEEE Systems Journal, vol. 5, no. 1 , pp. 61-69, Mar. 2011.

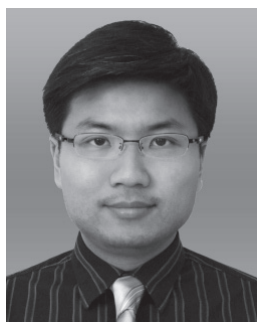

Chun-Hao Lo (S'08) received the B.S. degree in Electrical and Computer Engineering from the Ohio State University, Columbus, $\mathrm{OH}$ in 2003, the M.S. degree in Engineering from the University of Detroit Mercy, Detroit, MI in 2004, and the M.S. degree in Telecommunication from New Jersey Institute of Technology (NJIT), Newark, NJ in 2006. He is currently pursuing his Ph.D. at NJIT. His research interests include Smart Grid system, wireless adhoc, mesh, sensor, and personal area networks, as well as MAC and routing protocols.

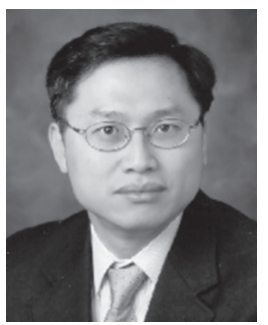

Nirwan Ansari (S'78-M'83-SM'94-F'09) received the B.S.E.E. (summa cum laude with a perfect gpa) from the New Jersey Institute of Technology (NJIT), Newark, in 1982, the M.S.E.E. degree from University of Michigan, Ann Arbor, in 1983, and the Ph.D. degree from Purdue University, West Lafayette, IN, in 1988. He joined NJIT's Department of Electrical and Computer Engineering as Assistant Professor in 1988, tenured and promoted to Associate Professor in 1993, and has been Full Professor since 1997. He has also assumed various administrative positions at NJIT. He authored Computational Intelligence for Optimization (Springer, 1997, translated into Chinese in 2000) with E.S.H. Hou, and edited Neural Networks in Telecommunications (Springer, 1994) with B. Yuhas. His current research focuses on various aspects of broadband networks and multimedia communications. He has also contributed over 350 technical papers, over one third of which were published in widely cited refereed journals/magazines. $\mathrm{He}$ has also guest-edited a number of special issues, covering various emerging topics in communications and networking. He is an IEEE Fellow (Communications Society), and has visited Chinese University of Hong Kong, Tohoku University of Japan, and National Cheng Kung University of Taiwan.

$\mathrm{He}$ was/is serving on the Editorial/Advisory Board of eight journals, including as a Senior Technical Editor of IEEE Communications Magazine (2006-2009). He had/has been serving the IEEE in various capacities such as Chair of IEEE North Jersey COMSOC Chapter, Chair of IEEE North Jersey Section, Member of IEEE Region 1 Board of Governors, Chair of IEEE COMSOC Networking TC Cluster, Chair of IEEE COMSOC Technical Committee on Ad Hoc and Sensor Networks, and Chair/TPC Chair of many conferences/symposia. He has been frequently invited to deliver keynote addresses, distinguished lectures, tutorials, and talks. Some of his recent recognitions include IEEE Leadership Award (2007, from Central Jersey/Princeton Section), the NJIT Excellence in Teaching in Outstanding Professional Development (2008), IEEE MGA Leadership Award (2008), the NCE Excellence in Teaching Award (2009), a Thomas Alva Edison Patent Award (2010), and designation as an IEEE Communications Society Distinguished Lecturer (2006-2009, two terms). 